\title{
BLIMPK/Streamline Surface Catalytic Heating Predictions on the Space Shuttle Orbiter
}

\author{
Jeremiah J. Marichalar*and William C. Rochelle ${ }^{\dagger}$ \\ ESCG/Jacobs Sverdrup, Houston, TX, 77058 \\ Benjamin S. Kirk ${ }^{\ddagger}$ and Charles H. Campbell ${ }^{\S}$ \\ NASA Johnson Space Center, Houston, TX, 77058
}

\begin{abstract}
This paper describes the results of an analysis of localized catalytic heating effects to the U.S. Space Shuttle Orbiter Thermal Protection System (TPS). The analysis applies to the High-temperature Reusable Surface Insulation (HRSI) on the lower fuselage and wing acreage, as well as the critical Reinforced Carbon-Carbon on the nose cap, chin panel and the wing leading edge. The object of the analysis was to use a modified two-layer approach to predict the catalytic heating effects on the Orbiter windward HRSI tile acreage, nose cap, and wing leading edge assuming localized highly catalytic or fully catalytic surfaces. The method incorporated the Boundary Layer Integral Matrix Procedure-Kinetic (BLIMPK) code with streamline inputs from viscous Navier-Stokes solutions to produce heating rates for localized fully catalytic and highly catalytic surfaces as well as for nominal partially catalytic surfaces (either Reinforced Carbon-Carbon or Reaction Cured Glass) with temperature-dependent recombination coefficients. The highly catalytic heating results showed very good correlation with Orbiter Experiments STS-2, -3, and -5 centerline and STS-5 wing flight data for the HRSI tiles. Recommended catalytic heating factors were generated for use in future Shuttle missions in the event of quick-time analysis of damaged or repaired TPS areas during atmospheric reentry. The catalytic factors are presented along the streamlines as well as a function of stagnation enthalpy so they can be used for arbitrary trajectories.
\end{abstract}

\section{Nomenclature}

\begin{tabular}{|c|c|}
\hline$\left(\hat{e}_{\xi}, \hat{e}_{\eta}, \hat{e}_{\zeta}\right)$ & streamline coordinate-aligned unit vectors \\
\hline$(\hat{i}, \hat{j}, \hat{k})$ & Cartesian-aligned unit vectors \\
\hline$(\xi, \eta, \zeta)$ & streamline coordinate system \\
\hline$(u, v, w)$ & Cartesian velocity components \\
\hline$(x, y, z)$ & Orbiter Cartesian axis system \\
\hline$\beta$ & chemical energy accommodation coefficient \\
\hline$r$ & position vector \\
\hline $\boldsymbol{V}$ & velocity vector \\
\hline$\epsilon$ & emissivity \\
\hline$\gamma$ & catalytic recombination rate coefficient \\
\hline$\lambda$ & thermal conductivity \\
\hline$\sigma$ & Stefan-Boltzmann constant $\left(0.1712 \times 10^{8} \mathrm{Btu} / \mathrm{hr}-\mathrm{ft}^{2}-\mathrm{R}^{4}\right)$ \\
\hline$b$ & bump factor, ratio of fully catalytic to nominal heating rat \\
\hline$C_{i}$ & mass fraction of $i^{\text {th }}$ species \\
\hline
\end{tabular}

*Aerothermal Engineer, Aerothermal and Flight Dynamics, 2224 Bay Area Blvd., JE 5WA

${ }^{\dagger}$ Project Manager, Aerothermal and Flight Dynamics, 2224 Bay Area Blvd., JE 5WC, Associate Fellow AIAA

$¥$ Aerospace Engineer, Applied Aerosciences and CFD Branch, 2101 NASA Pkwy., EG3

$\S$ Orbiter Aerothermal NASA Subsystem Engineer, Applied Aerosciences and CFD Branch, 2101 NASA Pkwy., EG3, Senior Member AIAA 


$\begin{array}{ll}D_{i j} & \text { multicomponent diffusion coefficient } \\ h^{o} & \text { enthalpy of formation } \\ H_{w} & \text { wall enthalpy } \\ h_{\text {arc }} & \text { heating parameter } \\ H_{c l} & \text { arc-jet nozzle centerline enthalpy } \\ M_{i} & \text { normal mass flux of } i^{\text {th }} \text { species } \\ P_{\text {meas }} & \text { measured test article surface pressure } \\ q & \text { heat flux per unit area } \\ T & \text { temperature } \\ t & \text { time } \\ (\mathbf{X o}, \mathbf{Y o}, \mathbf{Z o}) & \text { axial, lateral, and vertical Shuttle coordinates } \\ \mathrm{N} & \text { atomic nitrogen } \\ \mathrm{N}_{2} & \text { molecular nitrogen } \\ \mathrm{NO} & \text { nitric oxide } \\ \mathrm{O} & \text { atomic oxygen } \\ \mathrm{O}_{2} & \text { molecular oxygen } \\ \mathrm{x} / \mathrm{L} & \text { Orbiter axial distance from apex normalized by reference length }(\mathrm{L}=1290 \text { inches })\end{array}$

\section{Introduction}

$\mathrm{T}_{\mathrm{H}}^{\mathrm{H}}$

HE capability to analyze the catalytic heating effects of localized repaired or damaged surfaces on the Thermal Protection System (TPS) of the U.S. Space Shuttle Orbiter was a major recommendation set forth by the Columbia Accident Investigation Board ${ }^{1}$ before the Orbiter could be returned to flight. Because exposed underlying layers of material resulting from damage to the TPS surface can have different thermochemical properties from the Reaction Cured Glass (RCG) coating on the Orbiter windward acreage and/or the Reinforced Carbon-Carbon (RCC) on the wing leading edge, the chemical surface heating, or catalytic heating, occurring during Orbiter atmospheric reentry can significantly increase in the localized damage area beyond the values predicted for flow in chemical equilibrium. In addition, any repair to the TPS may cause a change in the chemical make-up of the repair site, which can also cause higher catalytic heating compared to the surrounding TPS. In order to incorporate localized catalytic heating into the Shuttle TPS thermal math models, catalytic heating amplification (or bump) factors will be used to increase the nominal heating rates to damaged or repaired areas.

In support of Return to Flight (RTF), the National Aeronautics and Space Agency (NASA) at Johnson Space Center (JSC) in Houston, TX requested that analyses be performed to assess the localized heating effects from highly catalytic surfaces that may be present on the Orbiter TPS during atmospheric re-entry. In the event that the Orbiter becomes damaged and must re-enter the atmosphere with TPS damage exposure or repair sites, a quick surface heating analysis must be performed to determine if aerothermal heating will become problematic. Because of the lack of catalytic material test data and because performing parametric analyses of catalytic heating solutions in localized areas with computational fluid dynamics (CFD) models is time and resource intensive, a modified two-layer approach consisting of streamline inputs derived from chemical non-equilibrium Navier-Stokes solutions and a boundary layer method was used to perform the analyses. The analyses were compared to the limited amount of catalytic flight data obtained from the Orbiter Experiments (OEX) program. ${ }^{2,3,4}$

The objective of the analyses was to ascertain whether the thermo-chemical kinetic gas model contained in the Boundary Layer Integral Matrix Procedure - Kinetic (BLIMPK) code $^{5}$ could be used to predict the catalytic heating rate overshoots exhibited in the OEX program flight measurements with temperaturedependent recombination coefficients from material data analyses of $\mathrm{RCG}^{6}$ and $\mathrm{C}_{742^{7}}$ coated tile. Early BLIMPK analyses used parametrically adjusted constant recombination rates to match the radiometer flight data at the Orbiter stagnation point. ${ }^{8}$ Later analyses for the Shuttle Entry Air Data System (SEADS) nose cap revealed that localized catalytic surfaces could produce heating rates that overshoot the theoretical fully catalytic (equilibrium) values. ${ }^{9}$ Additional observations of this overshoot phenomenon were also documented in other Shuttle analyses. ${ }^{10}$ If the modified two-layer method could be validated, it would be used to assess surface catalytic heating effects to damaged or repaired TPS locations on the Orbiter during atmospheric re-entry in support of RTF recommendations assuming a localized fully catalytic damage site. 


\section{Methodology}

The surface catalytic heating study presented in this paper was conducted over approximately two years and included research of historical flight data and analyses, arc-jet tests on coated and uncoated Hightemperature Reusable Surface Insulation (HRSI) tiles and RCC materials, a study of analytical approaches and a large number of new analyses with modified analytical methods and procedures. The following sections describe the progression of the catalytic heating study and the methods employed to develop a working analytical model for obtaining localized catalytic heating factors on a damaged or repaired Orbiter heat shield.

\section{A. Analysis of Flight Data}

The first study of catalytic heating factors for use on damaged or repaired locations on the Shuttle TPS began in late 2003. Because no measurements of surface catalysis had been obtained for the TPS repair materials or damaged TPS material, existing flight data from early Space Transportation System (STS) missions, which had highly catalytic C742 coatings on selected HRSI tiles, were used to obtain catalytic heating factors that could be applied to any region on the Orbiter. ${ }^{11}$ These C742 coatings were placed on two selected tiles along the Orbiter centerline for STS-2 and STS-3. STS-5 contained C742 coated tiles at six centerline locations and two wing locations as indicated in Figure $1 .^{2,3,4}$

During these early Shuttle flights, a unique phenomenon occurred when the flow passed from the partially catalytic RCG coated tiles to tiles with the C742 coating. The heating rates on the highly cat-

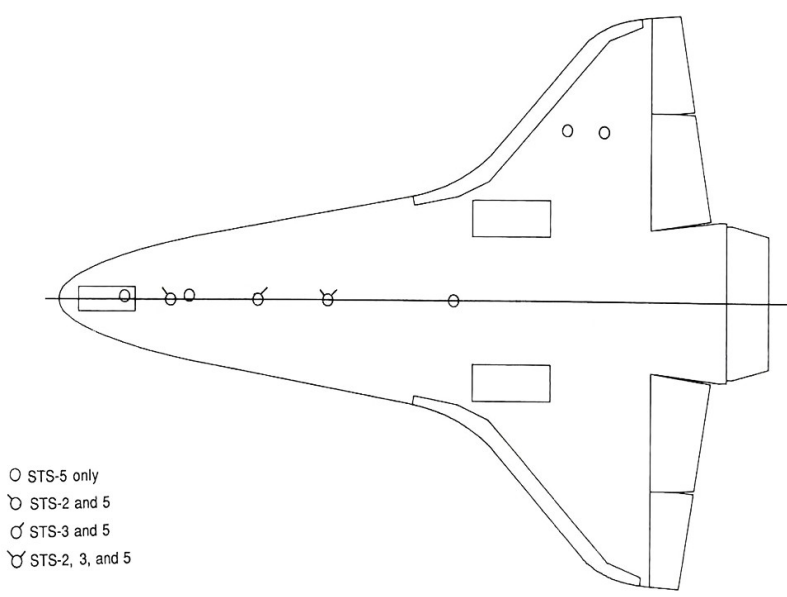

Figure 1. C742 catalytic tile locations on STS-2, -3 , and -5 . alytic tiles increased sharply above the partially catalytic tile values and even increased above the fully catalytic or equilibrium flow predicted values. The C742 coated-tile heating rates then drop gradually across these tiles and drop sharply again as the flow then passes over the next partially catalytic tile. Predicted values using the BLIMPK code for Flights STS-2, -3, and -5 have been documented that show this overshoot phenomenon. ${ }^{10,12,13,14,15}$ The overshoot is caused by a sudden drop in oxygen atom $(\mathrm{O})$ concentration at the edge of the highly catalytic surface, with a consequent sudden increase in the oxygen molecule $\left(\mathrm{O}_{2}\right)$ concentration. This causes a sudden release of dissociation energy and increased heating to the edge of the highly catalytic tile.

This phenomenon is not limited to tiles, but is documented for flow over the Orbiter RCC nose cap in which the SEADS pressure ports were installed. These ports were made of highly catalytic columbium metal. The sudden increase of chemical heating rate from the partially catalytic RCC surface as computed by the BLIMPK code over the columbium ports was explained by the sudden drop in atomic oxygen at the wall. After the flow passed the port, the oxygen atom concentration increased back to its nominal level over the RCC, and the heating was reduced to the lower catalytic value. The heating rate then alternately increased and decreased over each port in a saw-tooth fashion. ${ }^{9}$

The objective of this first catalytic heating study was to use the STS OEX flight data to determine catalytic heating factors by comparing the heating rates of HRSI tiles with the highly catalytic C742 coating to heating rates of the partially catalytic tiles with the original RCG coating. The study was based on the Development Flight Instrumentation data, ${ }^{2,3,4}$ which contained the measurements from STS-2, -3 , and -5 .

Recommended catalytic heating factors were defined for the Orbiter nose cap and chin panel region and the wing leading edge based on the catalytic factors derived from this study of STS-2, -3 and -5 OEX flight data. ${ }^{16}$ These factors were later used to perform thermal analyses on the Orbiter RCC panels by the Boeing thermal group that supports the Shuttle Orbiter LESS/RCC NASA System Engineer, Dr. D. M. Curry, of NASA JSC ES3. ${ }^{17}$ 


\section{B. Arc-Jet Data}

A search was made for previous (historical) data at the JSC arc-jet facility as well as for more recent data obtained in the past year. This section describes some of the data which was converted into catalytic factors for a range of enthalpies.

An assessment of wall temperature was made between coated and uncoated RCC test articles tested downstream of the exit of a conical nozzle in the JSC arc-jet Test Position 2 (TP2). ${ }^{18}$ This test data was generated in 1990-91 and was reported for uncoated $\mathrm{RCC}^{19}$ and for coated RCC. ${ }^{20,21}$ Unfortunately, the test conditions were not the same between the uncoated and coated test articles. However, the data for the coated articles can be extrapolated back to lower values of the heating parameter given below ${ }^{18}$

$$
h_{\text {arc }}=\sqrt{P_{\text {meas }}}\left(H_{c l}-H_{w}\right)
$$

Using this data, the measured surface temperatures were obtained from a laser pyrometer at two conditions of stagnation enthalpy for uncoated RCC and coated RCC. Catalytic (or bump) factors of 1.50-1.55 were calculated ${ }^{22}$ from the following equation, assuming the uncoated RCC and coated RCC emissivity were equal:

$$
b \equiv \frac{q_{\text {uncoated }}}{q_{\text {coated }}}=\frac{\sigma\left(\epsilon T^{4}\right)_{\text {uncoated }}}{\sigma\left(\epsilon T^{4}\right)_{\text {coated }}}=\frac{\left(T^{4}\right)_{\text {uncoated }}}{\left(T^{4}\right)_{\text {coated }}}
$$

Recent tests have been performed at the JSC arcjet facility in TP2 for uncoated or damaged RCC at higher enthalpies. The Pyrovision Infrared camera ${ }^{a}$ was used to obtain the distribution of surface temperatures on the test article as a function of time for these tests. An example of a temperature distribution on an RCC test article taken with the infrared camera during an arc-jet test is shown in Figure 2. In this case a fully catalytic damage site with exposed carbon substrate is located at the center of the test article. The results of these tests have not been published yet; however, preliminary results have been obtained ${ }^{\mathrm{b}}$. Because of the range of calculated stagnation enthalpies (from 9,500 to $13,000 \mathrm{Btu} / \mathrm{lb}$ ) and the variation of surface temperature during the tests, it was not possible to obtain a precise value of enthalpy for each catalytic factor. An extreme case of damaged (uncoated) RCC was

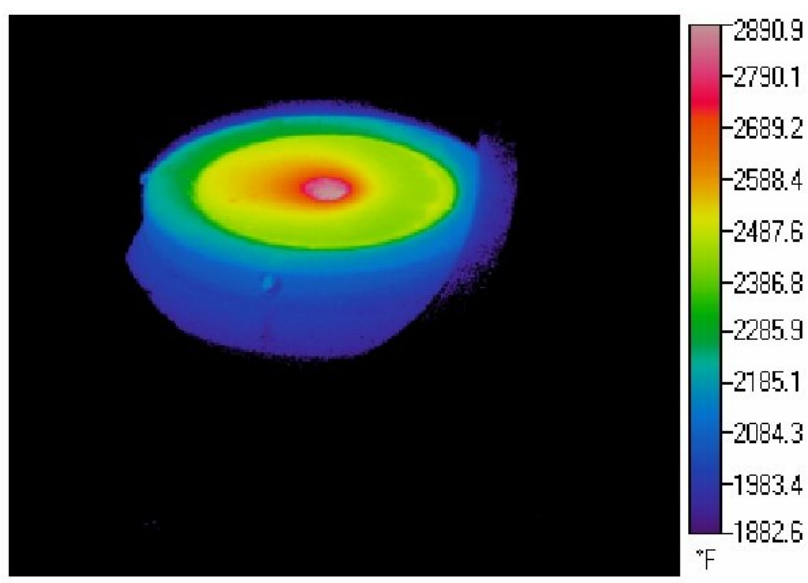

Figure 2. Temperature distribution on RCC test article from infrared camera during arc-jet test. performed recently at TP2 in which the calculated stagnation enthalpy was about 20,000 Btu/lb - significantly higher than any flight value for orbital return. The range of catalytic heating factors from evaluation of arc-jet data was determined to be $1.62-1.99{ }^{22}$

\section{Catalytic Recombination Coefficients}

In order to analyze the catalytic heating effects analytically, the recombination coefficients for the different surface materials must be defined and modeled correctly. Chemical heating occurs during atmospheric re-entry when the temperature of the air becomes large enough to excite and dissociate the air molecules into their respective constituents, namely oxygen and nitrogen. The catalytic heating depends on the recombination rates of the oxygen and nitrogen atoms of the dissociated air at the wall and their respective heats of formation. If the wall is fully catalytic, all of the atoms will recombine to become molecules at the wall, releasing the heat of dissociation and increasing the heating rate to the surface. For this case, the flow field in the vicinity of the surface will essentially be in chemical equilibrium. On the other hand, if the wall is non-catalytic to recombination, none of the atoms recombine to become molecules. In this case, the flow

\footnotetext{
${ }^{a}$ M9104 Mikron Pyrovision infrared camera (imaging pyrometer) manufactured by Mikron Infrared, Inc. of Oakland, NJ

${ }^{\mathrm{b}}$ Information transmitted from A. Rodriguez and R. Lewis/NASA-JSC to W. Rochelle/ESCG-Jacobs, 2005
} 
near the surface is essentially frozen at the particular atomic mass concentrations present at the edge of the boundary layer. This produces the lowest heating rate to the surface.

In reality, the surface of the HRSI tiles is neither non-catalytic nor fully catalytic, but partially catalytic. Hence, some of the atoms recombine at the wall, with resulting heating rates ranging between those experienced for a non-catalytic wall and a fully catalytic wall. The glassy outer RCG coating of the Orbiter HRSI tiles tends to have low catalycity compared to metallic surfaces such as copper or columbium which are nearly fully catalytic.

The recombination rates at the wall can be derived from the normal mass flux term in the Navier-Stokes equations for a gas in chemical non-equilibrium. ${ }^{23}$ Early analyses used a catalytic recombination speed, $k_{w}$, on the order of $700 \mathrm{~cm} / \mathrm{sec}$ for both oxygen and nitrogen recombination at the surface. However, later studies generated separate recombination rates for oxygen and nitrogen recombination based on experimental data. These rates can be determined as a function of wall temperature from data in arc-jet facilities for RCG coatings on HRSI tiles. ${ }^{6,24}$

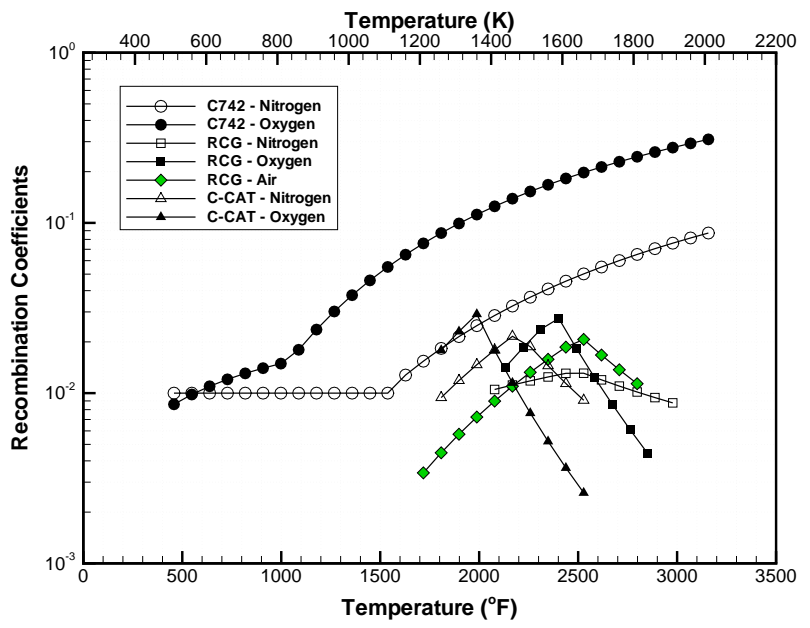

(a) Recombination coefficients vs. temperature.

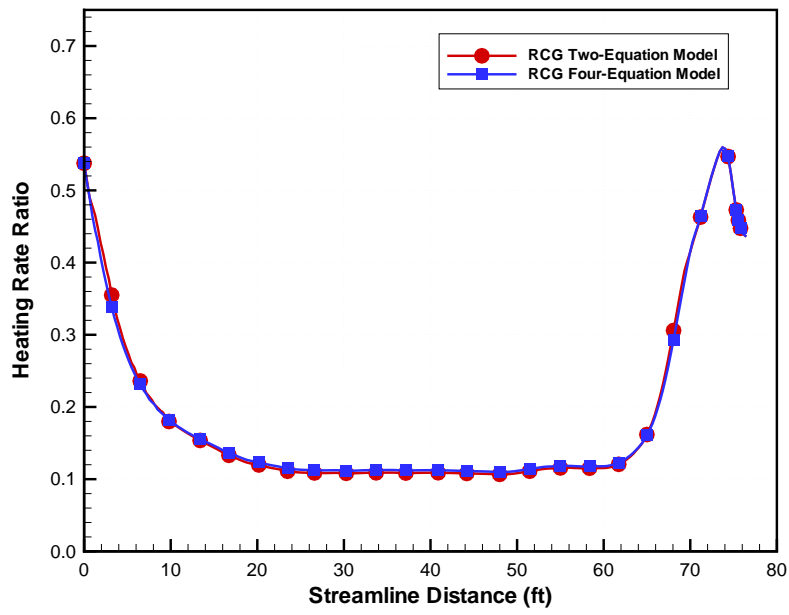

(b) Comparison between two and four-equation RCG recombination models.

Figure 3. Recombination coefficients and impact on heat transfer.

General expressions for recombination rates developed for RCG-coated tiles ${ }^{6}$ have been used in the literature for the predictions of surface heating rates for several years. These expressions for oxygen recombination rate coefficients, $\gamma_{\mathrm{O}}$, for two ranges of surface temperature are

$$
\begin{aligned}
\gamma_{\mathrm{O}} & =40 e^{-11440 / T_{w}} & & 1435 \mathrm{~K}<T_{w}<1580 \mathrm{~K} \\
& =39 \times 10^{-9} e^{21410 / T_{w}} & & 1580 \mathrm{~K}<T_{w}<1845 \mathrm{~K}
\end{aligned}
$$

The nitrogen recombination rate coefficients, $\gamma_{\mathrm{N}}$, for two ranges of surface temperature are

$$
\begin{aligned}
\gamma_{\mathrm{N}} & =6.1 \times 10^{-2} e^{-2480 / T_{w}} & & 1410 \mathrm{~K}<T_{w}<1640 \mathrm{~K} \\
& =6.1 \times 10^{-4} e^{5090 / T_{w}} & & 1640 \mathrm{~K}<T_{w}<1905 \mathrm{~K}
\end{aligned}
$$

Combined, these expressions make up the two-equation temperature-dependent model for the partially catalytic recombination coefficients for the HRSI RCG-coated tiles. The two-equation temperature-dependent nitrogen and oxygen recombination coefficients for RCG $,{ }^{6} \mathrm{C} 742,{ }^{7}$ and $\mathrm{RCC}$ (C-CAT), ${ }^{25}$ as well as the twoequation temperature-dependent air recombination coefficient model for $\mathrm{RCG}^{25}$ are plotted vs. temperature in Figure 3(a). Although a four-equation model for RCG partially catalytic recombination coefficients is available, the two-equation model was used in this analysis for consistency with previous analyses. A comparison between the four-equation and two-equation models for RCG recombination coefficients was performed to understand the differences in the two models. An example plot for this comparison is shown in Figure 3(b) 
for a streamline out to the Orbiter wing leading edge. The results of the comparison revealed that the difference between the models is insignificant.

A large database of temperature-varying recombination rates was generated in arc-jets and side-arm reactors at NASA Ames Research Center several years ago. ${ }^{25}$ Equations (3-6) as well as other equations in this database have been used in boundary layer programs such as the BLIMPK code, ${ }^{5}$ and in CFD programs such as the Langley Aerothermodynamic Upwind Relaxation Algorithm ${ }^{26}$ (LAURA) and the General Aerodynamic Simulation Program (GASP) $\operatorname{code}^{27}$ to predict heating rates on partially catalytic surfaces.

\section{Standard Two-Layer Methodology}

The general two-layer methodology is an inviscid/viscous analysis consisting of an inviscid solution for the free-stream flow to the edge of the boundary layer and a viscous boundary layer analytical procedure to calculate the flow properties and heating effects from the edge of the boundary layer to the surface. The boundary layer analysis for this catalytic heating study was conducted with the BLIMPK code. ${ }^{5}$ In past analyses with the BLIMPK code, the streamline inputs needed from the inviscid solution were usually extracted from inviscid Euler solutions. In a preliminary analysis, ${ }^{28}$ the BLIMPK code was used with the streamline inputs extracted from an existing inviscid Euler solution obtained from the Inviscid Equilibrium Code in 3-Dimensions (IEC3D). ${ }^{29,} 30$ The streamlines were extracted along the Orbiter centerline for Mach 12 and 18 in the STS-2 trajectory with the Axisymmetric Analog Two-Layer Convective Heating (AA2LCH) procedure. ${ }^{31}$ Figure 4 shows the flight data compared to the BLIMPK analysis for partially catalytic RCG

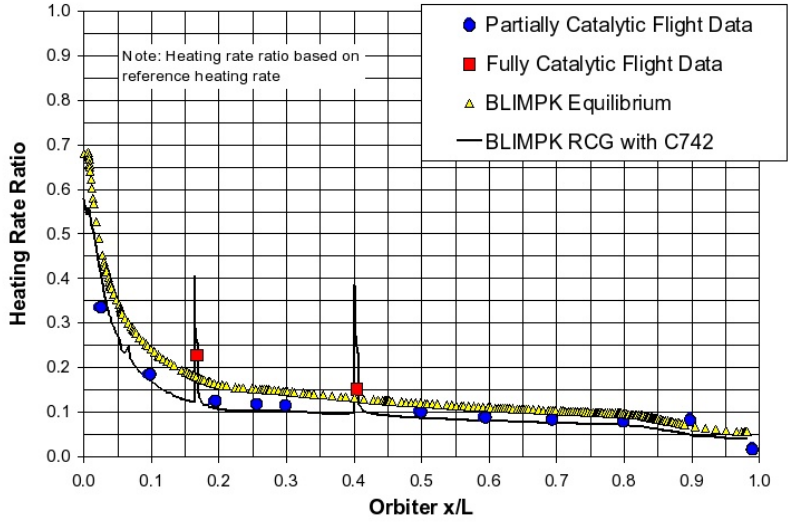

(a) Heating rate ratio for Orbiter centerline.

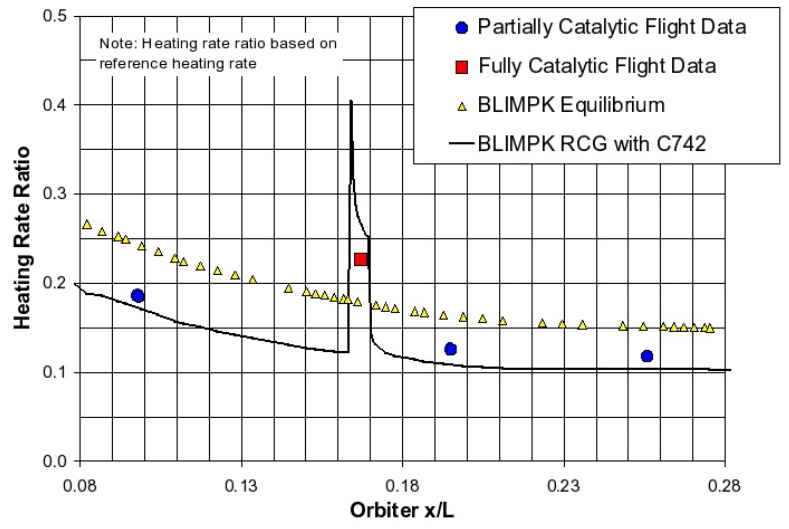

(b) Magnified.

Figure 4. Standard BLIMPK/IEC3D two-layer methodology compared to OEX flight data for Mach 18 in the STS-2 trajectory.

with C742 tiles and for full equilibrium for Mach 18. The forward most catalytic location is magnified in Figure 4(b). Because the preliminary BLIMPK analysis verified the thermo-chemical model in the BLIMPK code could satisfactorily model the catalytic heating overshoot observed along the centerline in the OEX flight data, NASA JSC requested that further analyses be conducted to model the catalytic heating effects observed on the Orbiter in the STS-2, -3 and -5 trajectories. However, since inviscid Euler solutions have largely been supplanted by computational fluid dynamics (CFD) Navier-Stokes solutions, a new method for obtaining the streamline inputs to BLIMPK was needed.

The catalytic heating analysis presented in this paper was performed with a modified two-layer approach rather than the general two-layer method. Similar to the general two-layer approach, the modified two-layer approach consists of the boundary layer solution with the BLIMPK code, but uses extracted streamline inputs from three-dimensional Navier-Stokes (viscous) solutions at the edge of the boundary layer instead of inviscid Euler solutions. The streamline inputs are extracted with a streamline mapping algorithm called SRFTRACE ${ }^{32}$ described in more detail in the next section. The SRFTRACE algorithm is similar in nature to the AA2LCH procedure used to extract streamline inputs from the inviscid Euler solutions and calculates the same streamline inputs needed for the BLIMPK code including streamline distance, metric coefficient, pressure distribution and entropy gradient. However, the SRFTRACE code is also capable of extracting 
the boundary layer edge species mass fractions from chemical non-equilibrium Navier-Stokes solutions, a capability not present in the AA2LCH code. With these streamline inputs, a non-equilibrium, non-isentropic analysis with defined boundary layer edge species mass fractions can be performed with BLIMPK.

\section{E. Modified Two-Layer Methodology}

In order to perform the catalytic heating analysis with the modified two-layer approach, three analytical tools were utilized in the following order: the Data-Parallel Line Relaxation (DPLR) CFD code, ${ }^{33}$ the SRFTRACE code, and the BLIMPK code.

\section{1. $\quad D P L R$}

The DPLR CFD code $^{33}$ was used to compute laminar Navier-Stokes solution at discrete trajectory points (time, altitude, and Mach number) for the Orbiter. All of the streamlines analyzed for the BLIMPK catalytic heating analysis originate from DPLR solutions. DPLR employs a thermo-chemical model and uses the fourequation temperature-dependent model for RCG for the entire windward acreage. Orbiter grid models exist for a low resolution (1-inch spacing between grid points) and a high resolution (0.25-inch spacing between grid points) computational analysis. ${ }^{34}$ It is possible to analyze localized catalytic heating analyses for the Orbiter with the DPLR code, but it is time and resource intensive. Because catalytic heating analyses may be needed at discrete points while the Orbiter is in-flight, the time needed to complete a localized catalytic heating analysis with DPLR can be restrictive. Additionally, this time requirement precludes the use of DPLR for rapid turnaround, large-scale parametric studies like those considered in the present work. However, DPLR laminar solutions for RCG partially catalytic tiles exist for a number of trajectory points from which streamline inputs can be extracted. All of the DPLR solutions are for a nominal re-entry angle of attack of $40^{\circ}$ and range between Mach numbers of 6 and 25 for the STS- 107 trajectory. ${ }^{35}$ The simulations employed a 5-species chemical non-equilibrium, thermal equilibrium model. DPLR-calculated heating rates are compared with the BLIMPK-calculated heating rates in section III. All of the streamline inputs for BLIMPK were extracted from the DPLR Navier-Stokes solutions with the SRFTRACE code.

\section{SRFTRACE}

The SRFTRACE code was used to map the data needed from the three-dimensional Navier-Stokes simulations into the format required for the BLIMPK code. The principal data inputs required for BLIMPK are the boundary-layer edge properties, the spatial location of the surface points, and the streamline metric coefficient. These first two input sets are trivially obtained from the CFD volume solutions, but the computation of the streamline metric coefficient warrants further explanation.

Figure 5 shows a representative streamline on the lower surface of the Orbiter. The reference coordinate system is denoted by the $(x, y, z)$ axes, which is the coordinate system used in the CFD solver. The $(\xi, \eta, \zeta)$ streamline coordinate system is introduced to provide the mapping for BLIMPK. In this curvilinear

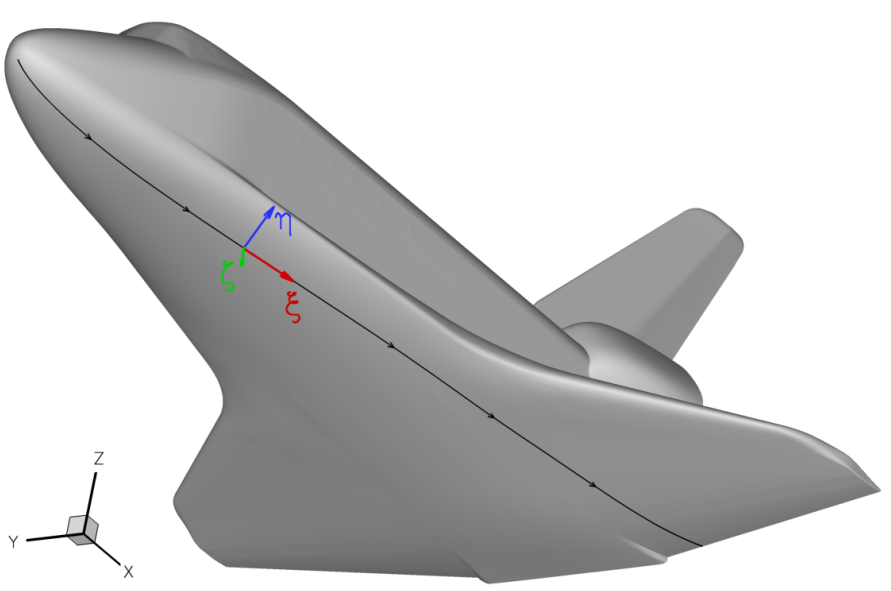

Figure 5. Reference and streamline coordinate systems. coordinate system the $\xi$-axis is tangential to the streamline and the $\zeta$-axis is normal to the surface of the vehicle. The $\eta$-axis completes the system.

An arbitrary location can be expressed in the reference Cartesian coordinates as

$$
\boldsymbol{r}=x \hat{i}+y \hat{j}+z \hat{k}
$$


Furthermore, points on the surface of the vehicle can also be defined as

$$
\boldsymbol{r}=\xi \hat{e}_{\xi}+\eta \hat{e}_{\eta}
$$

The unit vector $\hat{e}_{\eta}$ may be expressed as

$$
\left|\frac{\partial \boldsymbol{r}}{\partial \eta}\right| \hat{e}_{\eta}=\frac{\partial \boldsymbol{r}}{\partial \eta}
$$

where the magnitude term on the left hand side of the equation denotes the metric coefficient required by BLIMPK. This quantity measures the effective radius of curvature of the vehicle surface in the direction normal to the streamline.

Consider the identity

$$
\frac{\partial}{\partial t}\left(\frac{\partial \boldsymbol{r}}{\partial \eta}\right)=\frac{\partial}{\partial t}\left[\frac{\partial x}{\partial \eta} \hat{i}+\frac{\partial y}{\partial \eta} \hat{j}+\frac{\partial z}{\partial \eta} \hat{k}\right]
$$

which, upon rearranging the order of differentiation in the right-hand-side, becomes

$$
\frac{\partial}{\partial t}\left(\frac{\partial \boldsymbol{r}}{\partial \eta}\right)=\frac{\partial}{\partial \eta}\left[\frac{\partial x}{\partial t} \hat{i}+\frac{\partial y}{\partial t} \hat{j}+\frac{\partial z}{\partial t} \hat{k}\right]=\frac{\partial}{\partial \eta}[u \hat{i}+v \hat{j}+w \hat{k}]=\frac{\partial \boldsymbol{V}}{\partial \eta}
$$

The last term on the right-hand-side may be expanded using the following identity

$$
\frac{\partial()}{\partial \eta} \equiv \frac{\partial x}{\partial \eta} \frac{\partial()}{\partial x}+\frac{\partial y}{\partial \eta} \frac{\partial()}{\partial y}+\frac{\partial z}{\partial \eta} \frac{\partial()}{\partial z}
$$

Using (12) in (11) yields the following system of ordinary differential equations

$$
\frac{\partial}{\partial t}\left(\frac{\partial \boldsymbol{r}}{\partial \eta}\right)=\frac{\partial \boldsymbol{r}}{\partial \eta} \cdot \nabla \boldsymbol{V}
$$

which defines an initial-value problem for the metric coefficient distribution along a given streamline. At the stagnation point of the vehicle the nose radius is known and defines the initial value of the metric coefficient. An explicit time integration technique is used to compute the metric coefficient at downstream locations, similar to the approach outlined in Reference 31.

This procedure is implemented in a $\mathrm{C}^{++}$code called SRFTRACE. This tool uses the libMesh ${ }^{\mathrm{c}}$ opensource finite element library to read the surface and boundary-layer edge parameters, along with the surface grid. The surface grid is treated as a hybrid-element unstructured mesh, which removes any constraints on the topology of the original grid. The standard piecewise linear Lagrange basis functions are used for interpolation along the surface. The boundary-layer thickness and edge properties are located using the curvature method described in Reference 36.

The user specifies a point of interest on the surface and a streamline is generated via explicit integration in the direction opposite of the boundary-layer edge velocity. This procedure terminates in the upstream direction when the stagnation point is located. The streamline may also be extended downstream some user-specified distance if desired. The resolution of the points along streamlines depends on the resolution of the Navier-Stokes solution. In this study, the highest-resolution grids currently available for Orbiter RTF reentry ${ }^{34}$ simulations were used, providing a streamwise point distribution on the order of 0.25 inches. At each point along the streamline the tool interpolates a number of values from the CFD data including (i) surface pressure, (ii) species mass fractions at the edge of the boundary layer, (iii) total energy, (iv) surface normal, and (v) boundary layer edge velocity. The velocity gradient is also computed, as is entropy. In the current implementation, the entropy is computed assuming local equilibrium using the curve fits by Tannehill. ${ }^{37}$ It would be preferable to interpolate the entropy directly from the CFD solutions as well, but this is not supported with the existing post-processing tools. The tool outputs a table including all the information needed by BLIMPK, and optionally can produce a Tecplot ${ }^{\circledR \mathrm{d}}$ binary surface file including all the derived parameters for inspection.

\footnotetext{
${ }^{\mathrm{c}}$ http://libmesh.sourceforge.net

dhttp://www.tecplot.com
} 


\section{3. $B L I M P K$}

The bulk of the catalytic heating analysis, including calculation of the catalytic factors, was performed with the BLIMPK code, a FORTRAN based code developed by Aerotherm for NASA for use in aeroheating analyses during the Apollo program. The BLIMPK code was used in this analysis to calculate localized catalytic heating at points along centerline streamlines and streamlines out to the wing tile acreage and wing leading edge. The BLIMPK model, described in the latest User's Guide, ${ }^{5}$ incorporates temperaturedependent catalytic models and utilizes 15 nodes across the boundary layer. Subsequent modifications allow the catalytic model to be updated at each station along the streamline, as well as the ability to model 2,500 streamline stations. Macros and scripts have been used to speed up the pre-processing of the BLIMPK input files for large parametric studies.

Table 1. Freestream and stagnation properties for STS-5 and STS-107 Best-Estimate Trajectories.

\begin{tabular}{lccccc}
\hline Flight & $\begin{array}{c}\text { Time } \\
\text { From EI } \\
(\mathbf{s e c})\end{array}$ & $\begin{array}{c}\text { Altitude } \\
(\mathbf{f t})\end{array}$ & $\begin{array}{c}\text { Mach } \\
\text { No. }\end{array}$ & $\begin{array}{c}\text { Stagnation } \\
\text { Enthalpy } \\
(\mathbf{B t u} / \mathbf{l b m})\end{array}$ & $\begin{array}{c}\text { Stagnation } \\
\text { Pressure } \\
(\mathbf{a t m})\end{array}$ \\
\hline \hline STS-5 & 810.0 & 208,560 & 18 & 6,886 & 0.054392 \\
STS-107 & 375.8 & 248,430 & 25 & 11,839 & 0.019555 \\
STS-107 & 602.4 & 230,907 & 23 & 10,324 & 0.034366 \\
STS-107 & 916.3 & 203,819 & 18 & 6,830 & 0.050651 \\
STS-107 & 993.1 & 192,972 & 16 & 5,619 & 0.082356 \\
STS-107 & 1339.6 & 130,740 & 6 & 1,016 & 0.142274 \\
\hline
\end{tabular}

The BLIMPK code input was set up to model a chemically non-equilibrium boundary layer of 5 species with finite gas reaction rates for non-isentropic flow around a body. The inputs also included streamline distance, metric coefficient, pressure distribution, boundary layer edge species mass fractions, and entropy gradient. In addition, BLIMPK required stagnation enthalpy and pressure values which were extracted from the Best-Estimated Trajectory for STS- $5^{4}$ and STS- $107,{ }^{35}$ the two trajectories used in this analysis. These stagnation values are provided in Table 1.

\section{F. Orbiter Locations Investigated}

One of the goals of this analysis was to determine how well non-equilibrium, non-isentropic BLIMPK results based on a modified two-layer methodology would match the STS-2, -3 and 5 OEX flight data. Because the STS-5 mission had six nearcenterline $\mathrm{C} 742$ catalytic tiles in addition to the two wing C742 catalytic tiles, the STS-5 Best-Estimate Trajectory was utilized for a major part of the present analysis. In order to verify that the BLIMPK analysis was suitable for predicting the catalytic overshoot phenomena encountered during Orbiter atmospheric reentry, all of the centerline C742 catalytic sensors were investigated, as well as the two C742 coated wing tiles on STS-5. The thermocouple locations for the centerline sensors and two wing sensors on the Orbiter used for this analysis are listed in Table 2. ${ }^{2,3,4}$ Validating the current approach with these flight data allowed the methodology to be applied to the Orbiter nose cap, lower fuselage acreage, wing acreage, and wing leading edge with confidence.
Table 2. STS-5 data sensor locations.

\begin{tabular}{lccc}
\hline $\begin{array}{l}\text { Sensor } \\
\text { Label }\end{array}$ & $\begin{array}{c}\text { Xo } \\
\text { (in) }\end{array}$ & $\begin{array}{c}\text { Yo } \\
\text { (in) }\end{array}$ & $\begin{array}{c}\text { Zo } \\
\text { (in) }\end{array}$ \\
\hline \hline V09T9341A & 267.7 & 0.0 & 306.1 \\
V07T9452A $^{\diamond}$ & 361.5 & -5.2 & 285.6 \\
V07T9463A $^{\diamond}$ & 450.4 & 0.0 & 278.6 \\
V07T9464A $^{\diamond}$ & 486.5 & -6.4 & 277.4 \\
V09T9381A & 565.0 & -4.3 & 275.6 \\
V07T9468A $^{\diamond}$ & 620.1 & 0.0 & 274.4 \\
V07T9471A $^{\diamond}$ & 755.9 & 0.0 & 271.4 \\
V09T9521A & 878.9 & -12.8 & 268.8 \\
V07T9478A $^{\diamond}$ & 1002.0 & 0.0 & 266.0 \\
V07T9481A & 1129.3 & 0.0 & 263.2 \\
V07T9487A & 1265.0 & 0.0 & 260.2 \\
V07T9489A & 1392.4 & 0.0 & 262.8 \\
V07T9492A & 1511.3 & 0.0 & 274.8 \\
V07T9689A & 1222.6 & 280.3 & 283.9 \\
V07T9692A & 1294.1 & 277.9 & 282.0 \\
\hline
\end{tabular}

$\diamond$ Denotes C742 Coated Tile

* Denotes C742 Coated Wing Tile 


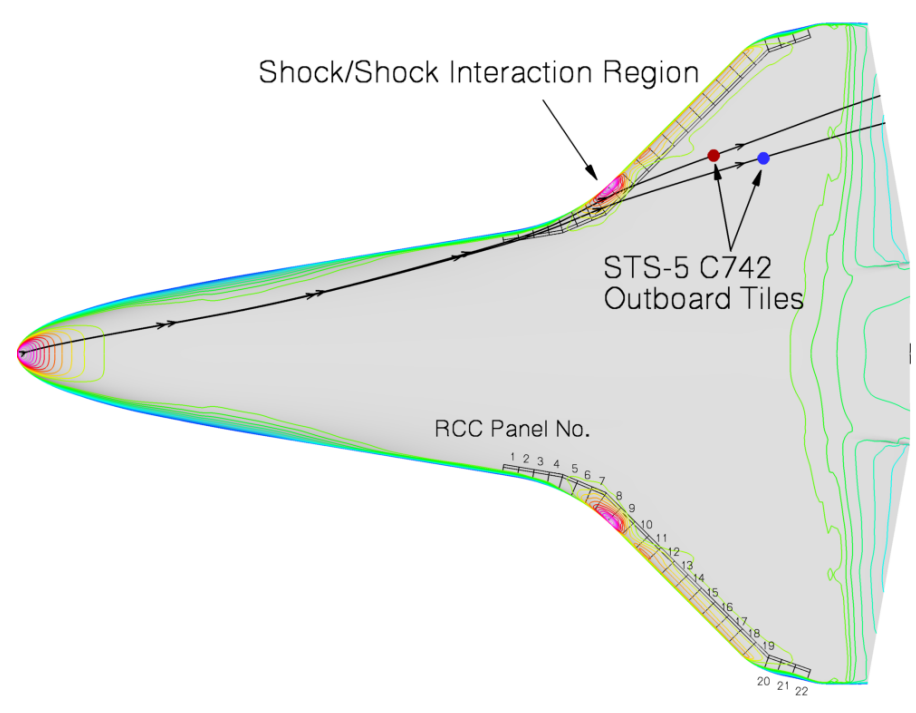

Figure 6. Surface pressure contours and streamlines to wing C742 tiles. RCC panel locations shown for reference.

The STS-5 BLIMPK wing analysis was compared to the STS-5 C742 highly catalytic flight data measurements mainly to judge the ability of the modified two-layer methodology to predict catalytic heating jumps on streamlines out to locations on the Orbiter wing. The two streamlines to the catalytic tile locations on the underside of the left wing on STS-5 pass very close to the bow shock/wing shock interaction region on the wing leading edge (in the vicinity of RCC panels 8 and 9), as shown in Figure 6. In past analyses, there have been problems running BLIMPK with the adverse pressure gradient that occurs in the shock/shock interaction region. ${ }^{38}$ This current analysis proves that BLIMPK can handle the large adverse pressure gradients which occur along the streamline near the shock/shock interaction region on the wing leading edge, provided that the appropriate boundary-layer edge properties are used. One observation from the current analysis is that it is critical to account for entropy swallowing in the presence of adverse pressure gradients when using this modified two-layer methodology.

The other purpose of this document is to present the predictions of the BLIMPK surface catalytic heating analyses for fully catalytic repair/damage locations at specific RCC sites including the nose, chin panel, and wing leading edge. The present BLIMPK surface catalytic heating analysis was performed for two RCC nose cap body point locations - one on the windward side and one on the leeward side - and one chin panel location. Streamlines for three panels with six zones per panel for the STS-107 trajectory were investigated, each passing through a respective body point on the wing leading edge of the Orbiter. A schematic of the wing leading edge panel showing the zone locations is shown in Figure 7(a). ${ }^{\mathrm{e}}$

Table 3 contains the body point locations on the nose, chin panel and wing leading edge for the RCC catalytic heating analysis. The body points for panels 7 and 9 in Table 3 are taken along a normal cut to wing leading edge. Figure $7(b)^{\mathrm{f}}$ shows the panel 9 normal cuts in detail with the body point locations labeled. The body points for panel 11 are taken along a planar cut in X-direction at constant value of $\mathrm{Y}$ at 60 percent semi-span.

The RTF BLIMPK analysis for these cases was performed to determine fully catalytic heating bump factors at different locations on the Orbiter RCC nose, chin panel and wing leading edge for specific times (Mach numbers) in the STS-107 trajectory. The BLIMPK-predicted catalytic factors for the RCC critical regions were calculated $^{28}$ for comparison to the original catalytic factors derived from the OEX flight data sensors for the STS-2, -3 and -5 C742 highly catalytic tiles. ${ }^{11}$

\footnotetext{
e Information transmitted from M. Fields/Boeing Houston to W. Rochelle/ESCG-Jacobs, 2005

${ }^{\mathrm{f}}$ Information transmitted from M. Barnwell/Boeing Houston to W. Rochelle/ESCG-Jacobs, 2005
}

\begin{tabular}{|c|c|c|c|c|c|}
\hline Panel & Zone & $\begin{array}{l}\text { Body } \\
\text { Point }\end{array}$ & $\begin{array}{l}\text { Xo } \\
\text { (in) }\end{array}$ & $\begin{array}{c}\text { Yo } \\
\text { (in) }\end{array}$ & $\begin{array}{c}\text { Zo } \\
\text { (in) }\end{array}$ \\
\hline $\mathrm{n} / \mathrm{A}$ & $\mathrm{n} / \mathrm{A}$ & 114 & 255.3 & 0.0 & 312.3 \\
\hline $\mathrm{n} / \mathrm{A}$ & $\mathrm{n} / \mathrm{A}$ & 1000 & 236.0 & 0.0 & 338.5 \\
\hline $\mathrm{n} / \mathrm{A}$ & $\mathrm{n} / \mathrm{A}$ & 1020 & 267.1 & 0.0 & 310.0 \\
\hline 7 & 1 & 5115 & 1067.5 & 199.6 & 280.7 \\
\hline 7 & 2 & 5113 & 1059.3 & 209.9 & 284.7 \\
\hline 7 & 3 & 5105 & 1052.4 & 218.5 & 289.8 \\
\hline 7 & 4 & 5100 & 1051.2 & 220.1 & 293.8 \\
\hline 7 & $5 \mathrm{~A}$ & 5155 & 1052.4 & 218.5 & 300.6 \\
\hline 7 & 6 & 5163 & 1059.3 & 209.9 & 312.8 \\
\hline 9 & 1 & 5514 & 1105.6 & 246.5 & 285.2 \\
\hline 9 & 2 & 5512 & 1101.4 & 250.6 & 287.0 \\
\hline 9 & 3 & 5505 & 1095.0 & 256.5 & 291.9 \\
\hline 9 & 4 & 5500 & 1093.6 & 257.6 & 294.5 \\
\hline 9 & $5 \mathrm{~A}$ & 5553 & 1094.3 & 256.4 & 300.6 \\
\hline 9 & 6 & 5563 & 1102.8 & 246.9 & 311.5 \\
\hline 11 & 1 & 2609 & 1149.6 & 281.0 & 289.7 \\
\hline 11 & 2 & 2607 & 1143.9 & 281.0 & 290.5 \\
\hline 11 & 3 & 2602 & 1126.0 & 281.0 & 290.5 \\
\hline 11 & 4 & 2600 & 1117.0 & 281.0 & 296.6 \\
\hline 11 & $5 \mathrm{~A}$ & 4602 & 1126.0 & 281.0 & 307.1 \\
\hline 11 & 6 & 4607 & 1143.9 & 281.0 & 315.0 \\
\hline
\end{tabular}

Note: n/A denotes a nose cap body point location 


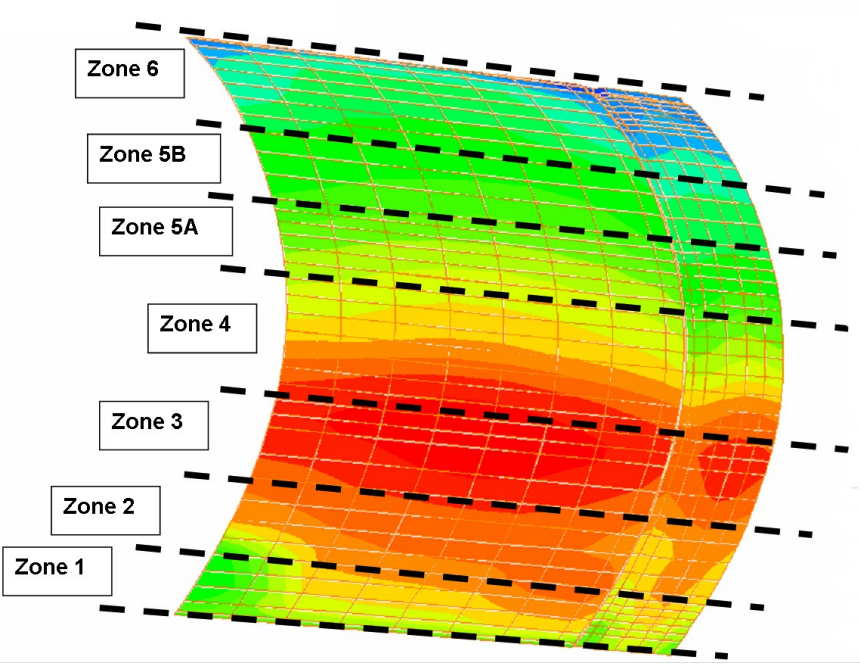

(a) Schematic of wing leading edge panel showing zone locations.

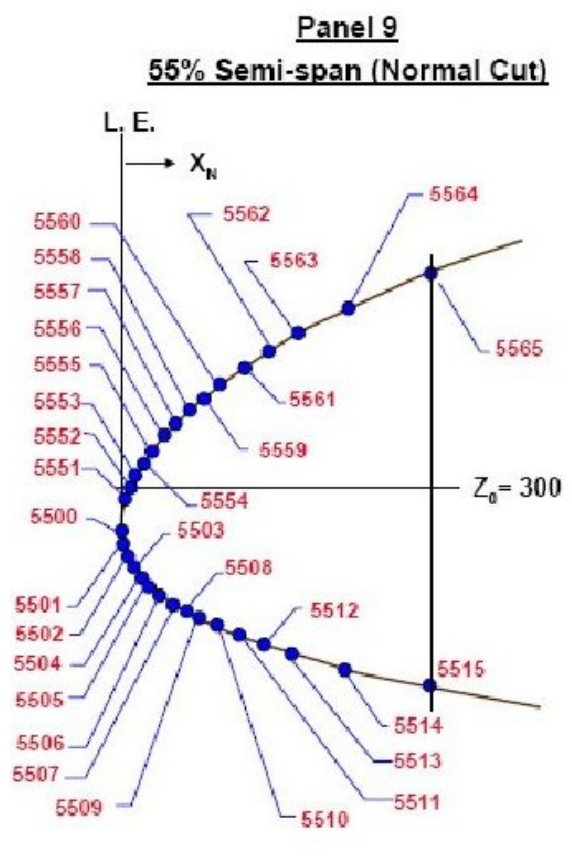

(b) Schematic of panel 9 showing normal cut on RCC wing leading edge and body point locations.

Figure 7. RCC panel geometry and body point locations.

\section{G. Assumptions and Cases Analyzed}

Several defining assumptions were made in order to perform the BLIMPK catalytic surface heating analysis. These assumptions are listed below:

1. Non-equilibrium, non-isentropic (Tannehill equilibrium entropy curve-fits ${ }^{37}$ ) solution with species mass fractions defined at the boundary layer edge (5 species model: $\mathrm{N}, \mathrm{O}, \mathrm{NO}, \mathrm{N}_{2}, \mathrm{O}_{2}$ )

2. NASA Ames Research Center two-equation temperature-dependent models for RCG, C742 and C-CAT (RCC) recombination coefficients from References 6, 7, and 25, respectively

3. Temperature-dependent RCG emissivity used for entire analysis ${ }^{7}$

4. C742 catalytic recombination coefficients for each catalytic tile location (approx. 8 inches long) on STS5 windward tile acreage analysis and fully catalytic recombination coefficients for each repair/damage location (approx. 6 inches long for nose and chin panel and 8 inches long for wing leading edge) for RCC analysis

5. Streamline cases for STS-5 trajectory at Mach 18 for the C742 highly catalytic tile locations (listed in Table 2)

6. Streamline cases for STS-107 trajectory at Mach numbers 25, 23, 18, 16, and 6 for body point locations in six zones at Panels 7, 9 and 11 (90 total cases)

The STS-2, -3 and -5 OEX flight data sensor locations are shown in Table 2, the body point locations for the STS-107 analyses are listed in Table 3, and the relevant stagnation properties for each trajectory point investigated are shown in Table 1. Catalytic location length and recombination coefficient variance sensitivity studies were conducted for selected cases. 


\section{Results}

The following section presents the results of the BLIMPK catalytic heating analysis performed on streamlines for selected points in the STS-5 (for comparison to the OEX flight data) and STS-107 trajectories. All of the heating rate results are presented in non-dimensional form as heating rate ratios based on a reference heating rate. The catalytic heating factors are derived in the same manner as they are in Reference 22, as the fully catalytic heating rate divided by the partially (nominal) catalytic heating rate. The centerline, nose cap and chin panel streamline analyses are evaluated first, and then the wing acreage and wing leading edge streamline analyses are evaluated.

\section{A. Lower Fuselage Centerline Region}

The catalytic heating effects from the six C742-coated tiles on the Orbiter windward centerline during the STS-5 mission were evaluated ${ }^{28}$ to verify that the BLIMPK code could model the catalytic heating overshoot observed on the STS-5 OEX flight data. Figures 8(a) and 8(b) show a Mach 18 comparison of the STS-5

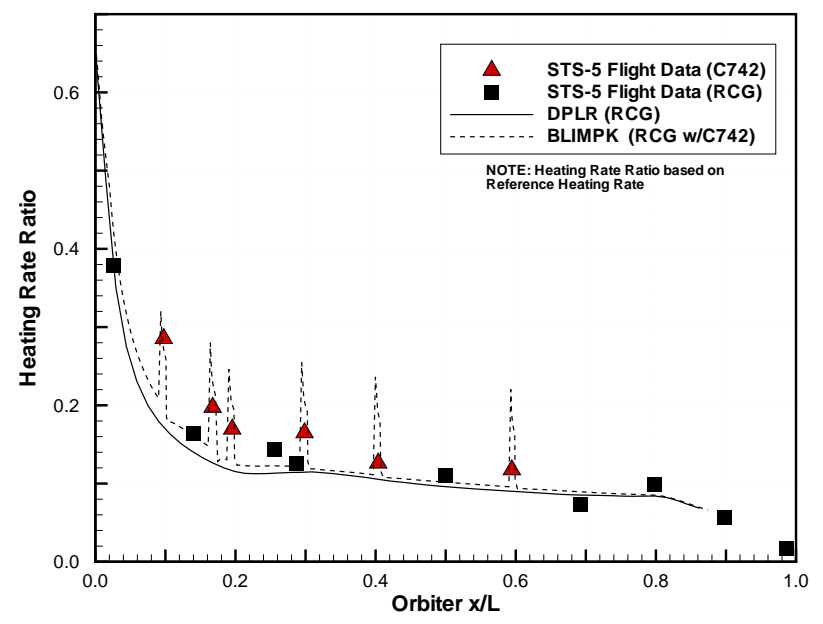

(a) Heating rate ratio for Orbiter centerline.

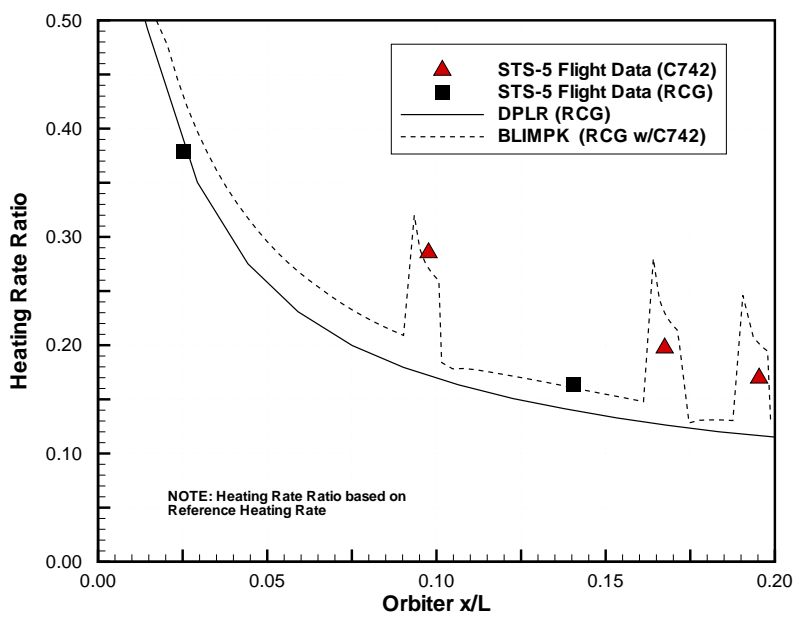

(b) Magnification.

Figure 8. DPLR and BLIMPK centerline analysis compared to STS-5 flight data for Mach 18.

OEX flight data and the BLIMPK calculated heating rate ratios for the centerline partially catalytic RCG tiles with highly catalytic C742-coating at sensor locations indicated in Table 2.

The entire streamline calculation and all the available centerline flight data is presented in Figure 8(a), and a magnification of the upstream C742 tile locations is shown in Figure 8(b). The flight data for the C742 catalytic locations is for the center of the 8-inch tile, where the BLIMPK catalytic heating has dropped significantly from the peak value at the upstream edge of the C742-coated tile. It is at this center location of each BLIMPK catalytic jump that the comparison with the STS-5 OEX flight data was made.

Figures $8(\mathrm{a})$ and $8(\mathrm{~b})$ show that the BLIMPK calculated heating rates compare very well with the STS-5 OEX flight data for the RCG and C742 coated tiles. The catalytic heating calculated by BLIMPK at the first C742 tile location slightly under predicts the STS-5 data value at that location (although it is well within the uncertainty of the data for this location at this point in the trajectory, $6.3 \%^{4}$ ), but the rest of the downstream BLIMPK catalytic heating jumps overshoot the STS-5 flight data. The BLIMPK overshoot for the downstream catalytic locations is expected because BLIMPK does not model the transient properties of the chemical heating occurring during reentry. The BLIMPK thermochemical model does not consider recombination of the atoms upstream. As the dissociated atoms travel downstream, they are partially exhausted by each highly catalytic tile location. Therefore, there are fewer atoms to recombine downstream at the other highly catalytic locations. Because there is more than one localized highly catalytic location along the centerline, this effect can be observed in the STS-5 flight data. 


\section{B. Nose Cap/Chin Panel Region}

A BLIMPK catalytic heating analysis was performed along centerline streamlines for the STS-107 trajectory to predict catalytic heating effects on the critical nose cap and chin panel $\mathrm{RCC}$ region. ${ }^{22}$ The analysis was conducted for leeward and windward streamlines for the nose RCC region of the Orbiter. The analysis was done with both C-CAT (RCC) and RCG partially catalytic models with fully catalytic repair/damage sites at body point 114 on the windward nose, body point 1000 on the leeward nose, and body point 1020 on the chin panel (cf. Table 3).

Figure 9 shows the Mach 18 BLIMPK heating rate ratio results of the windward streamline analysis with the DPLR predicted heating rate ratios included for comparison. The DPLR and BLIMPK calculated heating rate ratios compare very well for both the C-CAT and RCG analyses, but at the stagnation point, the BLIMPK C-CAT (RCC) result com-

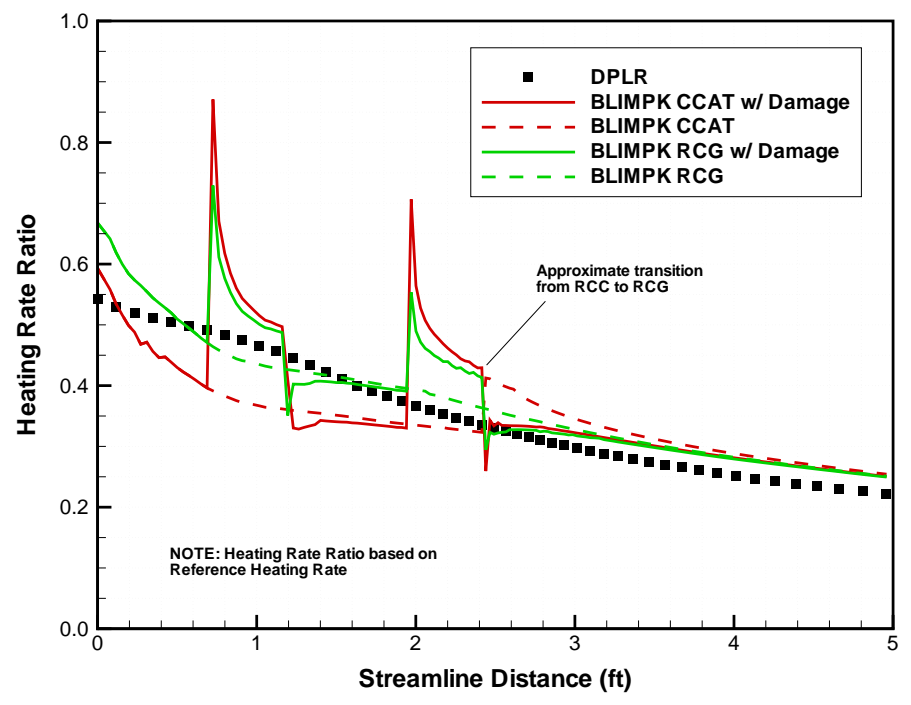

Figure 9. DPLR and BLIMPK windward centerline analysis for Mach 18 in the STS-107 trajectory. pares better with the DPLR result than the BLIMPK RCG result. The fully catalytic jumps from the C-CAT analysis are larger than the jumps from the RCG analysis. Because the partially catalytic heating rates from C-CAT are also lower than the RCG partially catalytic heating rates, the catalytic heating factors based on the C-CAT analysis are larger than the catalytic factors based on the RCG analysis. The BLIMPK C-CAT partially catalytic heating rate increases abruptly after the second fully catalytic jump because of the transition to the RCG HRSI tiles.

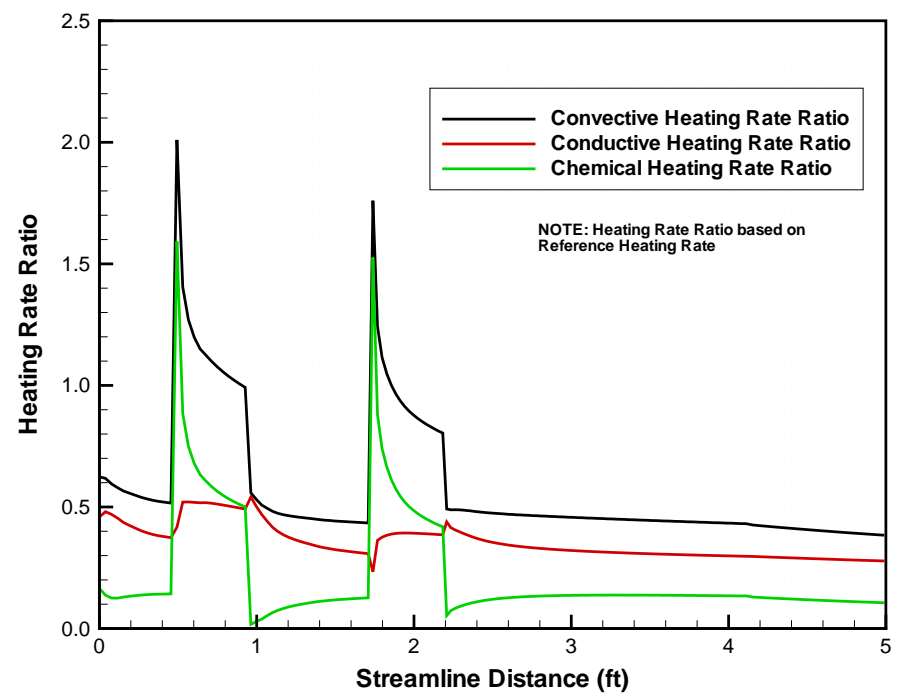

Figure 10. BLIMPK Mach 25 windward centerlines heating rate components.
The two fully catalytic jumps shown in Figure 9 were examined more closely in the Mach 25 windward centerline analysis. The convective heating rate (equivalent to the heating rate ratios presented in this paper) transferred to the surface, $q_{\text {conv }}$ is the sum of a conduction term, $q_{\text {cond }}$ and a chemical term, $q_{\text {chem }}$ :

$$
q_{\text {conv }}=q_{\text {cond }}+q_{\text {chem }}
$$

The convective heating rate contributions from the conductive and chemical heating rate terms are shown in Figure 10 for the BLIMPK Mach 25 windward centerline analysis. The conductive heating rate is the product of the thermal conductivity of the material and the temperature gradient at the surface. The chemical heating rate is computed from a combination of unequal thermal and multicomponent diffusion coefficients and species concentration gradients. ${ }^{23}$ If thermal diffusion is neglected, Equation (14) can be rewritten ${ }^{39}$

$$
q_{c o n v}=-\lambda\left(\frac{\partial T}{\partial \zeta}\right)+\sum_{i} \beta_{i} h_{i}^{o} M_{i}
$$


where the normal mass flux of the $i^{\text {th }}$ species, $M_{i}$, may be expressed as

$$
M_{i}=\rho \sum_{j} D_{i j}\left(\frac{\partial C_{j}}{\partial \zeta}\right)
$$

where the summation is over all species.

The chemical (or catalytic) heating occurs during hypersonic reentry and causes increased heating at the surface of the Orbiter. The air molecules (mostly molecular nitrogen and oxygen, or $\mathrm{N}_{2}$ and $\mathrm{O}_{2}$ ) are highly excited by the hypersonic shockwave and dissociate into their atomic constituents, $\mathrm{N}$ and $\mathrm{O}$, which may then react to form nitric oxide, NO. At the surface, the atoms may recombine to form molecules, releasing their dissociation energy in the form of heat which is then transferred into the surface. The rate of recombination determines the amount of chemical, or catalytic, heating. Some surfaces are very prone to massive recombination (highly catalytic materials), while some are deterrents to recombination (partially catalytic materials). By tracking the species mass fraction concentrations, the level of recombination, or catalycity can be observed.

Figure 11 exhibits the five species mass fraction concentrations on the Orbiter windward centerline at Mach 25 in the STS-107 trajectory. Between 0.0 and 0.5 feet and 1.0 and 1.7 feet, C-CAT recombination coefficients are being used for the surface heating calculations. In these areas, the species mass fraction concentrations appear nominal for hypersonic reentry. Oxygen is almost fully dissociated, shown by the high amount of oxygen atoms compared to the number of oxygen molecules. There are more nitrogen molecules than nitrogen atoms in the partially catalytic region because nitrogen has a higher excitation temperature, and therefore, does not dissociate as quickly as oxygen. At the fully catalytic damage sites, between 0.5 and 1.0 feet and 1.7 and 2.2 feet, there are significant increases in the $\mathrm{N}_{2}, \mathrm{O}_{2}$ and $\mathrm{NO}$ molecules, while there are also significant decreases in the amount of atomic nitrogen and oxygen. The increase in nitrogen and oxygen molecules shows that much more

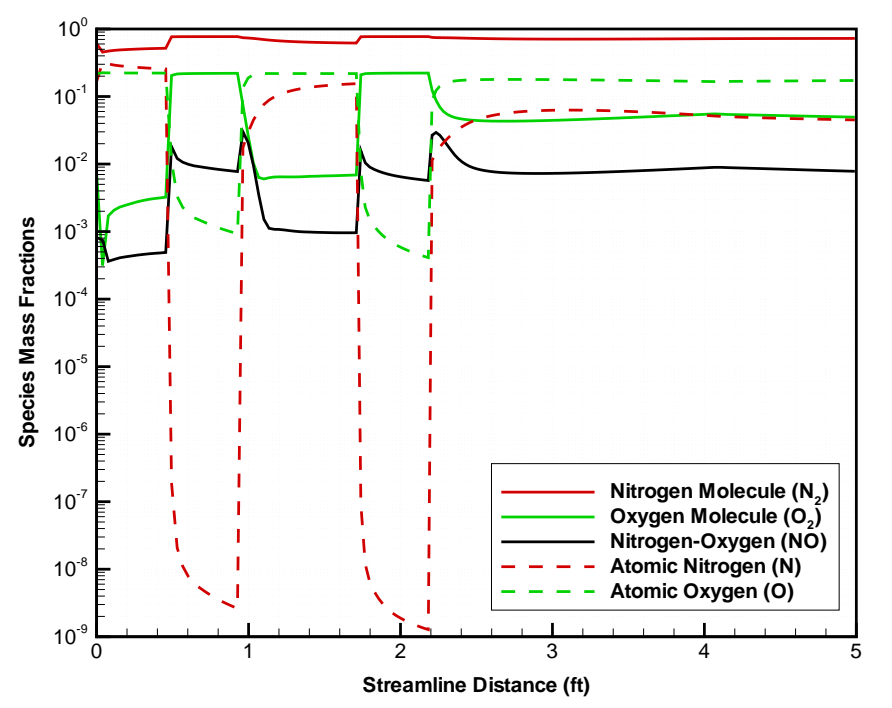

Figure 11. BLIMPK Mach 25 windward centerline species mass fractions.

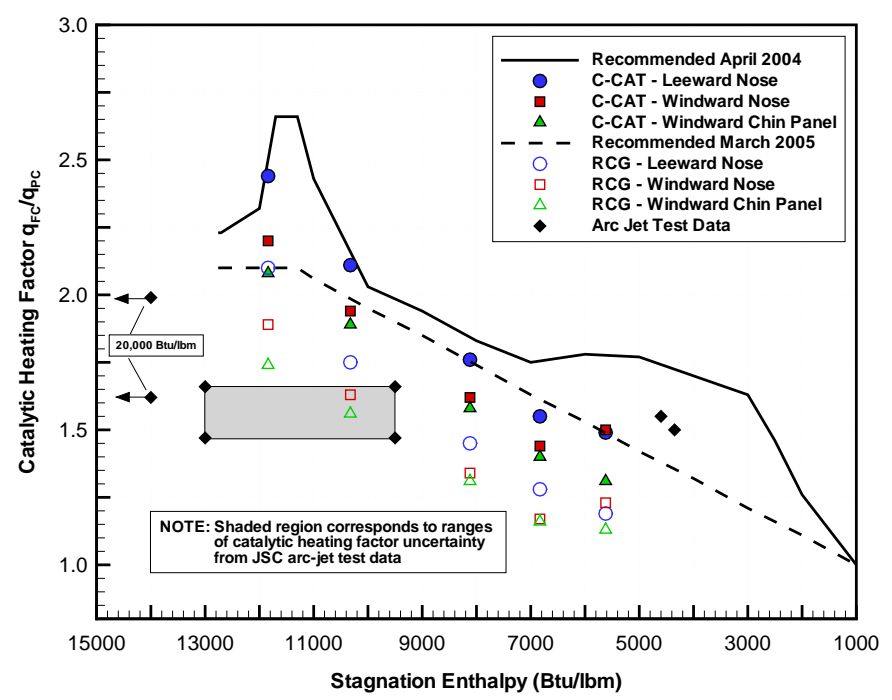

Figure 12. Catalytic factors derived from Orbiter centerline RCC nose cap analysis. recombination is occurring, hence the higher heating rates that can be seen in Figure 10. After 2.2 feet, the species mass fraction concentrations appear nominal once again, but are now higher than they were for the other partially catalytic regions because the RCG recombination coefficients were used for the surface heating calculations at this point.

The BLIMPK catalytic heating analysis along centerline streamlines for the STS-107 trajectory was performed to obtain catalytic heating factors on the critical nose cap and chin panel RCC region to be used in real-time thermal analyses for future Shuttle flights. ${ }^{22}$ Catalytic heating factors were calculated for fully catalytic repair/damage on the nose cap and chin panel region with RCG and C-CAT (RCC) partially 
catalytic heating rates at the trajectory points listed in Table 1 for STS-107. The RCG and C-CAT derived catalytic heating factors obtained from this analysis are plotted in Figure 12 along with the Recommended Catalytic Model for the Orbiter Nose Region proposed in April 2004 (based on the OEX flight data analysis in Reference 11) and in March $2005^{40}$ as an upper limit (based on the STS-107 analysis with BLIMPK). Arcjet test data points (described earlier) are also shown for comparison. As mentioned before, the catalytic factors predicted by BLIMPK based on the C-CAT partially catalytic heating rates are higher than the factors based on the RCG partially catalytic heating rates. In general, the catalytic factors obtained on the leeward side of the nose cap (where partially catalytic heating rates are lower) tend to be more severe than the catalytic factors obtained for the windward side. The catalytic factors also tend to decrease with decreasing enthalpy and as the damage location moves further away from the stagnation point.

\section{Wing Acreage Region}

A major focus of this BLIMPK catalytic surface heating analysis was the Orbiter wing tile acreage for STS-5. One of the major objectives of this analysis was to prove BLIMPK could successfully handle the large adverse pressure gradient induced by the the Orbiter bow-shock/wing leading edge-shock interaction and determine how well BLIMPK could model the catalytic heating overshoot observed in the flight data on the two C742 highly catalytic wing acreage tiles. ${ }^{41}$ Streamlines passing through these sensors are shown in Figure 6.

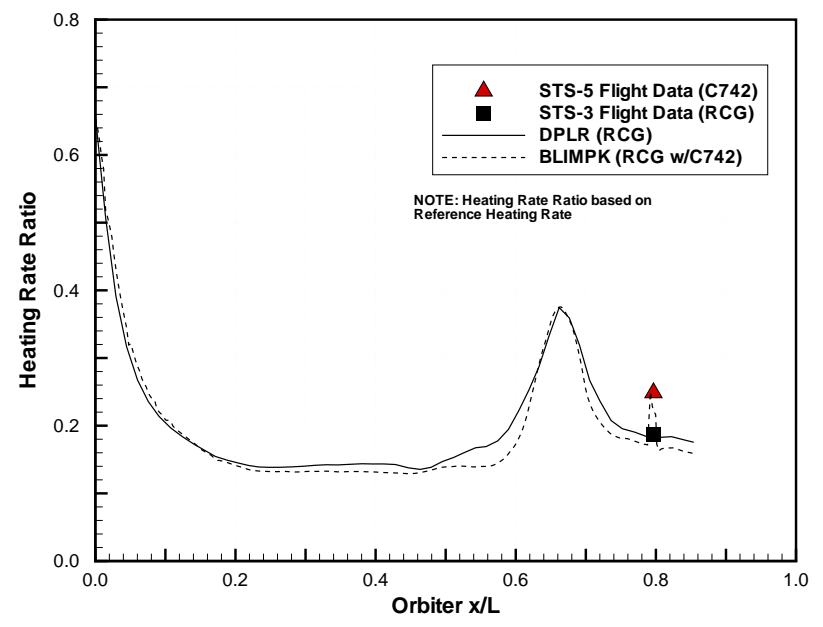

(a) Heating rate ratio for entire streamline.

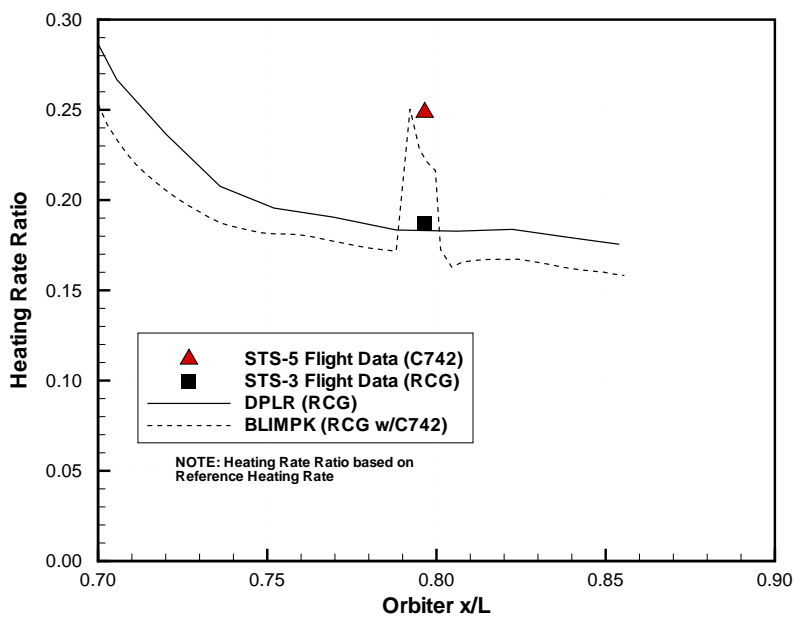

(b) Magnification.

Figure 13. DPLR and BLIMPK predicted heating rates for OEX sensor location V07T9689A at Mach 18 in the STS-5 trajectory.

The convective heating rate ratio results for the Mach 18 streamline out to the V07T9689A sensor on the Orbiter during STS-5 are shown in Figure 13(a) with the C742 catalytic jump at a streamline distance of approximately $85 \mathrm{ft}$. Both the DPLR solution heating rates and the BLIMPK solution heating rates are shown for comparison. STS-2 and -3 had very similar RCG partially catalytic heating rates at the sensor location, but only the heating rate at the sensor location for STS-3 is shown. The DPLR and BLIMPK generated heating rates are very close, and the BLIMPK solution was able produce the same peak heating rate in the adverse pressure gradient near the SSI region. Figure 13(b) shows the magnification of the V07T9689A sensor location along the STS-5 Mach 18 streamline. The DPLR heating rate ratio at the sensor location is slightly lower than the STS-3 partially catalytic heating rate ratio, while the BLIMPK partially catalytic heating rate ratio is even lower. The BLIMPK predicted value at the center of the C742 tile (location of the flight data measurement) comes very close to the STS- 5 flight data value. The BLIMPK prediction could possibly be higher if more data points were included across the tile, but it is still within the uncertainty of the flight data measurement $\left(6.3\right.$ percent $\left.^{4}\right)$.

The convective heating rate ratio results for the Mach 18 streamline out to the V07T9692A sensor on the Orbiter during STS-5 are shown in Figure 14(a) with the C742 catalytic jump at a streamline distance 
of approximately $90 \mathrm{ft}$. Both the DPLR solution heating rates and the BLIMPK solution heating rates are

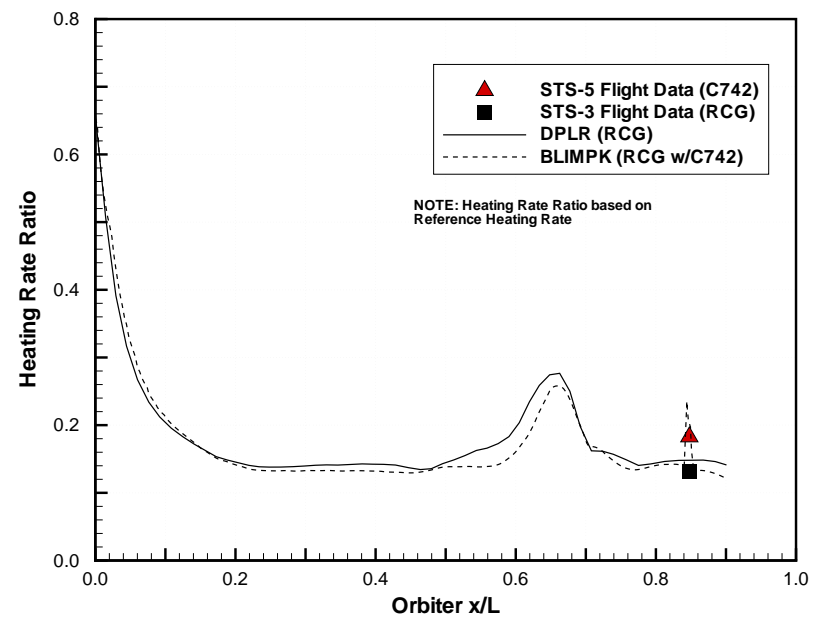

(a) Heating rate ratio for entire streamline.

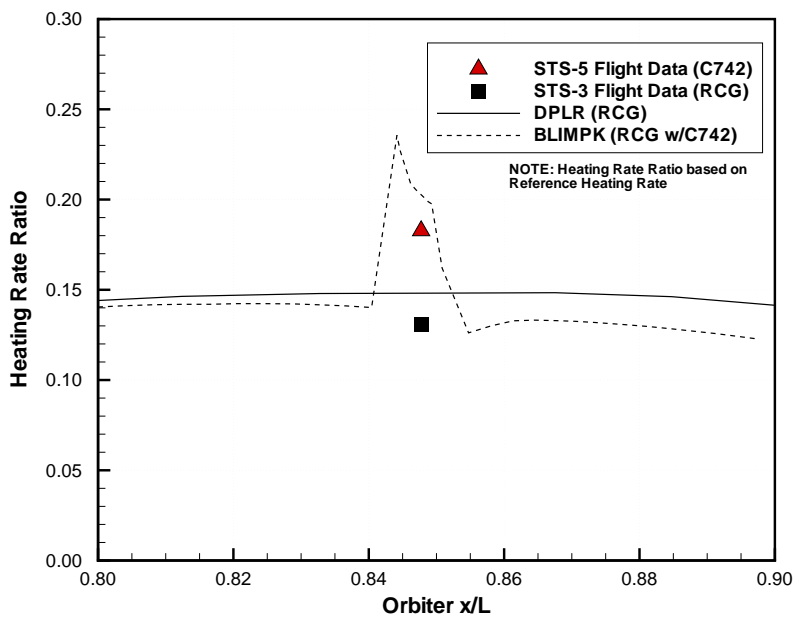

(b) Magnification.

Figure 14. DPLR and BLIMPK predicted heating rates for OEX sensor location V07T9692A at Mach 18 in the STS-5 trajectory.

shown for comparison. Again, although STS-2 and -3 had very similar RCG partially catalytic heating rates at the sensor location, only the heating rate at the sensor location for STS-3 is shown. The DPLR and BLIMPK generated heating rates are very close, but there is a larger difference between the peak heating rates in the two solutions at the adverse pressure gradient near the SSI region. Figure 14(b) shows the magnification of the V07T9692A sensor location. The DPLR heating rate at the sensor location is in close agreement with the STS-3 partially catalytic heating rate, while the BLIMPK partially catalytic heating rate is even closer. The BLIMPK predicted value at the center of the C742 tile is greater than the STS-5 flight data value even with the uncertainty of the flight data measurement (6.8 percent $\left.{ }^{4}\right)$.

Although the BLIMPK heating rate values for the V07T9689A sensor streamline are slightly lower than the flight data measurements at the sensor location, the BLIMPK derived highly catalytic to partially catalytic heating rate bump factors are higher. The BLIMPK calculated bump factor (1.39) is slightly higher than the factor computed from the STS-5 highly catalytic heating rate and the STS-3 partially catalytic heating rate (1.33) for the V07T9689A sensor location. The BLIMPK highly catalytic to partially catalytic heating rate bump factor for the V07T9692A sensor location is also slightly higher (1.47) than the value computed from the STS-5 highly catalytic heating rate and the STS-3 partially catalytic heating rate (1.4).

\section{Wing Leading Edge Region}

The purpose of the BLIMPK analysis ${ }^{41}$ along the off-centerline (wing) streamlines was to obtain catalytic heating factors for certain STS-107 trajectory points at locations on the RCC wing leading edge which could be applied in a thermal math model. The catalytic heating factors obtained from the new analysis would supplement the factors for the wing presented in Reference 11. Although 90 streamline cases were analyzed, the BLIMPK code was able to achieve converged solutions for only 85 of those cases. All streamlines for Panel 7, Zone 6 were unable to reach convergence, possibly due to the very deep expansion around the wing leading edge that occurs at that location.

Of the 85 converged solutions, the worst case heating rates and temperatures occur during Mach 23 in the STS-107 trajectory in the shock/shock interaction region on the wing leading edge at Panel 9, Zone 3. Figure 15(a) shows the worst case heating to the RCC wing leading edge at Panel 9, Zone 3 for Mach 23 in the STS-107 trajectory. The 8-inch fully catalytic damage location is shown at a streamline distance of approximately 75 feet. The BLIMPK predicted heating rate ratios are very close to those predicted by the DPLR CFD solution up until a distance of about 20 feet at which point the two solutions diverge to a maximum difference at the peak heating location on the RCC wing leading edge. The maximum difference in 
the heating between the BLIMPK and DPLR solutions occurs at the higher Mach numbers (the worst-case is Mach 25). The difference drops as the Mach number (or enthalpy) decreases. This difference may be because the current analysis assumes local equilibrium when computing the entropy gradient along the streamline as discussed previously. This approach should be most accurate near the stagnation point of the vehicle where the boundary-layer edge Mach number is low, and would become increasingly invalid as the flow accelerates rapidly around the vehicle, violating the assumption of local equilibrium.

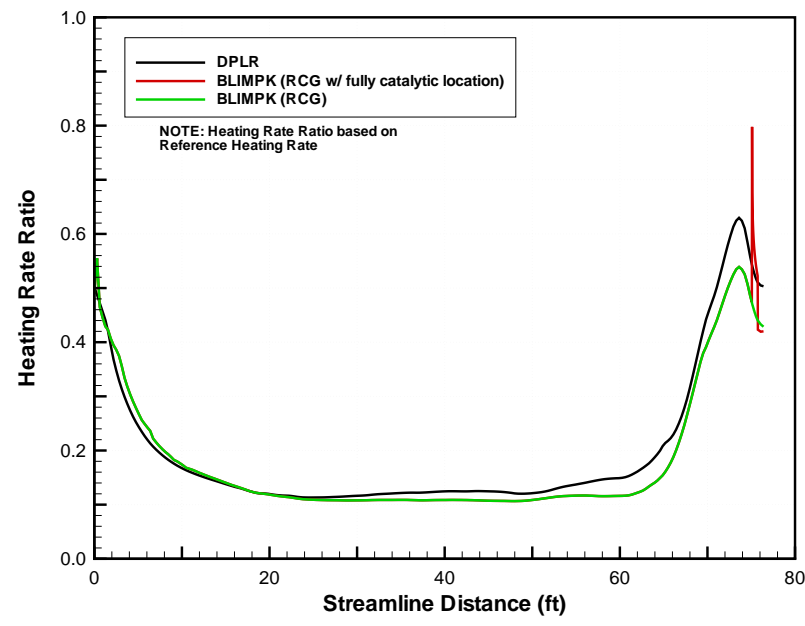

(a) Heating rate ratio for entire wing streamline.

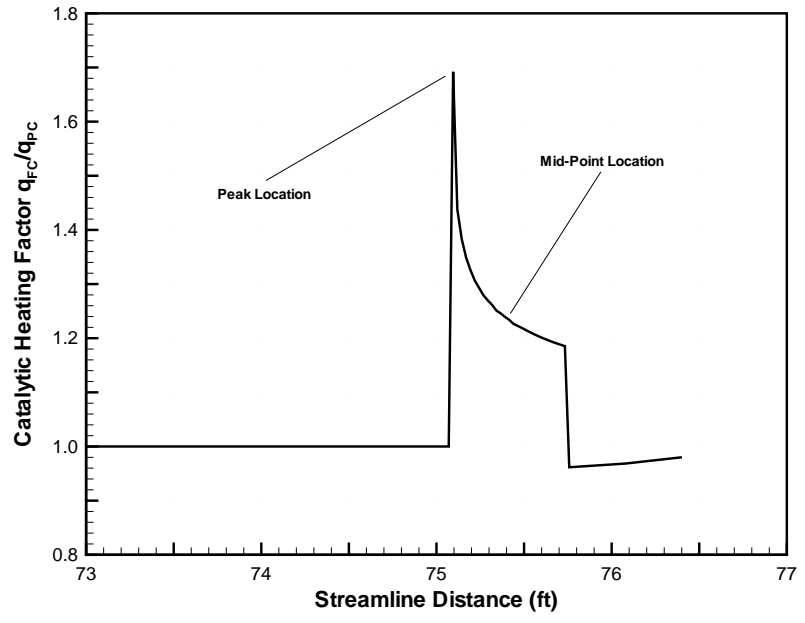

(b) Magnification.

Figure 15. DPLR and BLIMPK results for fully catalytic region at Orbiter RCC Panel 9 , Zone 3 at Mach 23 in the STS-107 trajectory.

Figure 15(b) shows a magnification of the fully catalytic damage site where the asymptotic peak and the exponential decay of the catalytic overshoot can be seen over the entire catalytic location. The peak catalytic factors and the catalytic factors at the mid-point were obtained for each converged solution and are presented in Reference 41. The real in-flight catalytic heating factor at the leading edge of the damage location is in between these two values. Although the worst-case heating rates and temperatures occur on Panel 9, Zone 3 (windward), the worst-case catalytic factors actually occur on the leeward zones (Zone 5A and 6), as will be discussed below.

Figure 16 displays the mid-point catalytic bump factors versus streamline distance in feet for all zones (for converged solutions) for Panel 11. Figure 16 shows that Zones 3 and 4 are closer to the apex of the wing leading edge and have the lowest catalytic factors, while the more windward and leeward zones have higher catalytic factors with the highest bump factor at either Zone 5A or 6 . The peak and midpoint catalytic bump factors for all converged cases along with the location and trajectory information and the fully catalytic and partially catalytic heating rates are contained in Reference 41. The Mach 6 cases were not evaluated as extensively as all fully catalytic bump factors were calculated to be unity, consistent with the negligible dissociation levels for this low freestream enthalpy (approximately 1,000 Btu/lbm, cf. Table 1).

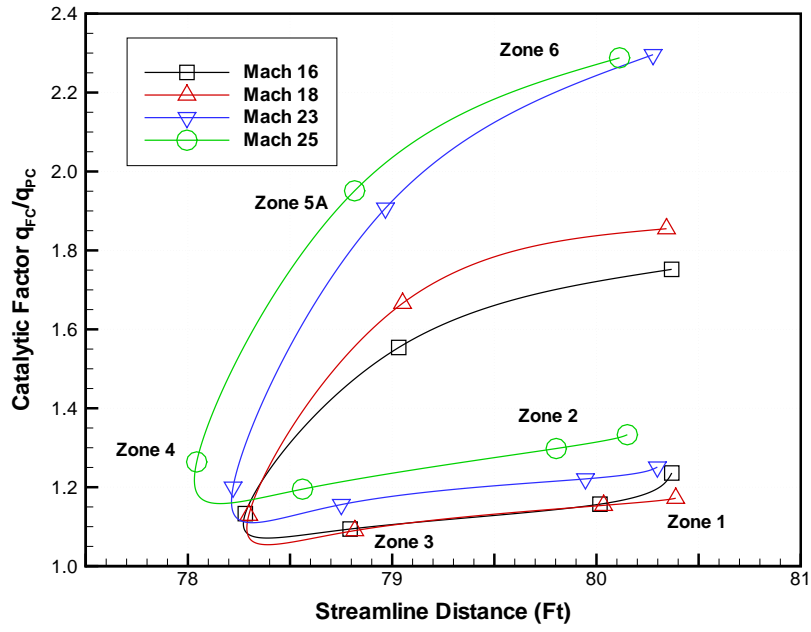

Figure 16. Mid-point catalytic factors for Panel 11. 
In Figure 17, the recommended catalytic heating factor model for the Orbiter wing region presented in Reference 11 is compared to the peak and mid-point catalytic factors predicted by BLIMPK in the present analysis for Zone 3 at Panels 7, 9 and 11, respectively. The recommended model was derived from the STS-5 C742 highly catalytic measurements on left wing tile locations and the STS-2 and -3 partially catalytic measurements at the same locations. The BLIMPK catalytic factor predictions for the two wing catalytic tiles on STS-5 match very well with the recommended model as do the peak catalytic factors at Panels 7 and 9 from the STS-107 trajectory. The mid-point catalytic factor predictions at all the panels in Zone 3 from the STS-107 trajectory are significantly lower as are the peak catalytic factors at Panel 11. A limited amount of arcjet catalytic data ${ }^{\mathrm{g}}$ obtained on a model placed

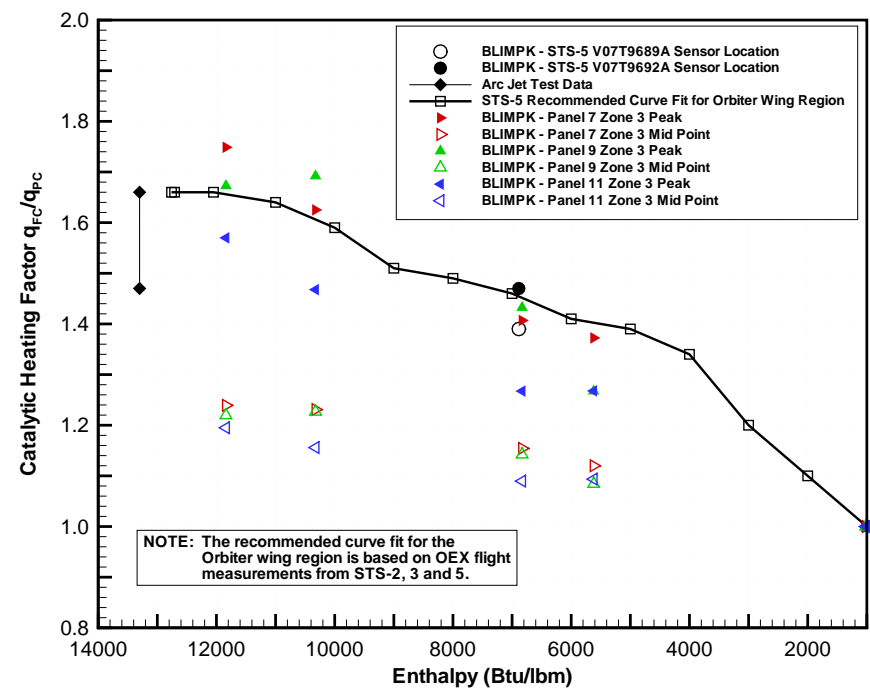

Figure 17. BLIMPK peak and mid-point wing leading edge catalytic factors compared to recommended model. in a wedge holder (flow parallel to surface) is also presented in Figure 17 for comparison with the BLIMPK predictions and the flight correlations. This range of arc-jet data resulted in factors of 1.47-1.66 based on a surface temperature increase of between 300$400{ }^{\circ} \mathrm{F}$, respectively, on the damaged RCC coating (assumed fully catalytic) compared to the undamaged RCC (partially catalytic). The analysis of these factors was the same as that reported in Reference, ${ }^{22}$ by taking the ratio of the fourth power of the temperatures and assuming the surface emissivity was the same for damaged and undamaged RCC. An undamaged RCC surface temperature of $2500{ }^{\circ} \mathrm{F}$ was used, and a computed arc-jet centerline (stagnation) enthalpy of 13,300 Btu/lb was determined.

\section{E. Sensitivity Study}

As noted in Reference 22, the catalytic heating factor is sensitive to many different parameters, and it is important to understand how those parameters affect the chemical heating. For the STS-107 analysis, catalytic location lengths were varied to determine if the size of a catalytic location had any effect on the overall bump factor. The recombination coefficients were also changed from RCG to C-CAT (RCC with Type-A Sealant) to observe the effect on the catalytic heating factors in the STS-107 wing leading edge analysis, although in this analysis, the flow would traverse RCG coated tiles from the stagnation point, then the RCC wing leading edge, and then the catalytic damage site. In an actual flight case, a wing leading edge streamline would start at the RCC nose stagnation point, then flow over the RCG HRSI tiles, and then back over the RCC wing leading edge and over the catalytic damage.

Figure 18(a) contains the heating rate ratio result for the sensitivity study on the length of the fully catalytic location for Panel 9, Zone 3 at Mach 18 in the STS-107 trajectory. Figure 18(a) reveals that changing the length of the fully catalytic damage site from 8 inches to 3 inches has no effect on the peak catalytic heating or bump factor. Because the length has changed, however, there is an effect on the midpoint catalytic heating rate and the corresponding bump factor. The new mid-point distance is only 1.5 inches from the peak for the 3 inch catalytic region and has a bump factor of approximately 1.24, while the mid-point of the 8 inch catalytic region is 4 inches from the peak and has a bump factor of 1.14.

Figure 18(b) shows the heating rate ratio result for the sensitivity study performed on varying the recombination coefficients between RCG and RCC (C-CAT) for Panel 9, Zone 3 at Mach 18 in the STS-107 trajectory. Figure 18(b) reveals that the RCC recombination coefficients yield a lower partially catalytic heating rate while generating a higher fully catalytic heating rate at the damage site as seen in the STS-107 RCC nose analysis in Figure 12. The higher fully catalytic heating rate and lower partially catalytic heating rate yield a larger catalytic bump factor (approximately 1.9 at the peak; 1.3 at the mid-point) than the RCG recombination coefficients (approximately 1.45 at the peak; 1.14 at the mid-point).

\footnotetext{
gInformation transmitted from A. Rodriguez and R. Lewis/NASA-JSC to W. Rochelle/ESCG-Jacobs, 2005
} 


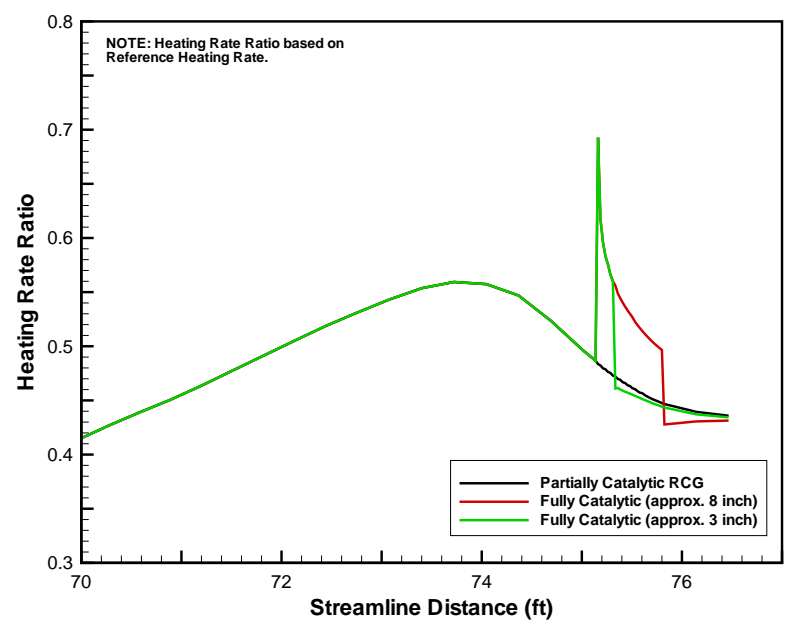

(a) Catalytic length sensitivity study results for STS-107 Mach 18 Panel 9, Zone 3.

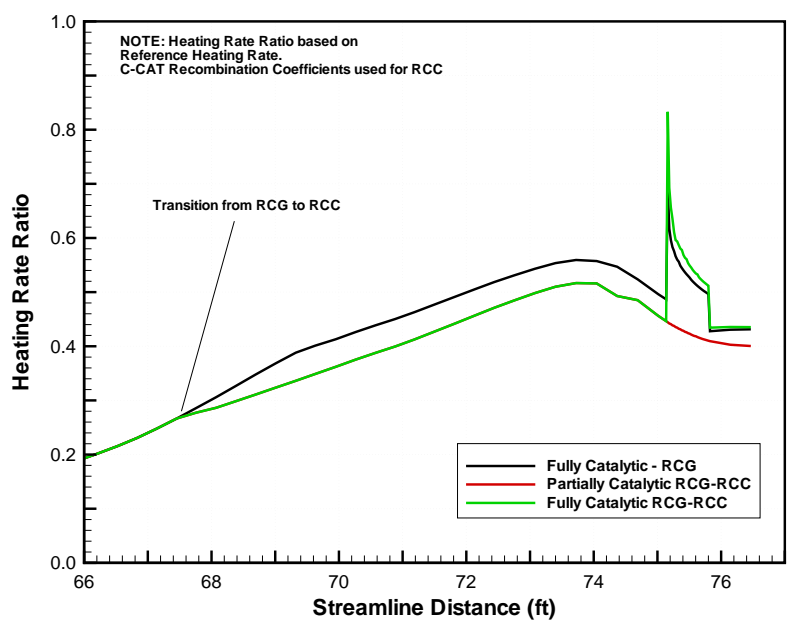

(b) Recombination coefficient sensitivity study results for STS-107 Mach 18 Panel 9, Zone 3.

Figure 18. Results from sensitivity studies.

\section{Conclusions}

This paper presented the methodology and results of a two-layer surface catalytic heating analysis on the Orbiter RCG windward tile acreage and critical RCC nose cap, chin panel and wing leading edge panels. Comparisons of the OEX STS-2, -3, and -5 flight data (with the highly catalytic C742 coatings) along the fuselage centerline and wing tile acreage were made with the BLIMPK heating predictions. The BLIMPK predicted catalytic heating jumps compared very closely to the catalytic heating jumps derived from the OEX flight data. This method was extended to the RCC regions to calculate heating jumps for localized fully catalytic locations at assumed points of repair or damage on the critical RCC.

The highest catalytic heating factors calculated for the RCC regions were slightly over 2.0 for the nose cap leeward side and on the order of 1.5 for the windward side. Catalytic heating factors computed at RCC Panels 7, 9, and 11 at six zones per panel for five free-stream Mach nos. were lowest (on the order of 1.2) in regions where the heating rate was the highest (Zones 3,4). The catalytic factors were the highest on the leeward side (Zones 5A,6) and at Zones 1 and 2 on the windward side where heating rates were lower.

The factors presented in this paper were recommended for use with potential repaired and damaged areas of RCC for real-time analysis for future flights in which in-flight repair would have to be made prior to reentry or flown as-is.

\section{Acknowledgments}

J. J. Marichalar and W. C. Rochelle thank S. A. Bouslog (TPS NASA Subsystem Engineer, JSC ES3) for inspiring the concept of using catalytic heating overshoots to analyze damaged or repaired TPS surfaces, D. M. Curry (JSC ES3) for long-term leadership in catalytic heating to Orbiter LESS surfaces, K. C. Wang (Boeing-Houston) for use of the AA2LCH code for early analysis of two-layer streamline inputs to BLIMPK, and J. A. Fredo (formerly of Lockheed Martin and ESCG) who scanned nearly all the STS-2, -3, -5 flight data and made numerous wing leading edge calculations and plots during the study. B. S. Kirk and C. H. Campbell thank D. Prabhu (Eloret Corp.) for solutions to the DPLR code used in the SRFTRACE/BLIMPK analyses and M. J. Wright (NASA Ames Research Center) for development of the DPLR code which has been used in numerous Orbiter reentry CFD analyses since the Columbia accident. 


\section{References}

${ }^{1}$ The Columbia Accident Investigation Board, "The CAIB Report - Volume I", http://caib.nasa.gov, August, 2003.

${ }^{2}$ Hartung, L. C. and Throckmorton, D. A., "Space Shuttle Entry Heating Data Book," Vol. I - STS-2, NASA RP 1191, Parts 1 and 2, 1988.

${ }^{3}$ Hartung, L. C. and Throckmorton, D. A., "Space Shuttle Entry Heating Data Book," Vol. I - STS-3, NASA RP 1192, Parts 1 and 2, 1988.

${ }^{4}$ Hartung, L. C. and Throckmorton, D. A., "Space Shuttle Entry Heating Data Book," Vol. I - STS-5, NASA RP 1193, Parts 1 and 2, 1988.

${ }^{5}$ Murray, A. L., "Further Enhancements of the BLIMP Computer Code and User's Guide," AFWAL-TR-88-3010, June 30,1988

${ }^{6}$ Kolodziej, P. and Stewart, D. A., "Nitrogen Recombination on High-Temperature Reusable Surface Insulation and the Analysis of its Effects on Surface Catalysis," Paper 87-1637 presented at AIAA $22^{\text {nd }}$ Thermophysics Conference, Honolulu, Hawaii, June 8-9, 1987

${ }^{7}$ Stewart, D. A., "Catalytic Surface Effects Experiment (CSE) for Space Shuttle," Orbiter Experiments (OEX) Aerothermodynamics Symposium, NASA CP 3248, Part, 1, April 1995, pp. 171-197

${ }^{8}$ Ting, P. C., Rochelle, W. C., and Curry, D. M., "Comparison of Viscous Shock Layer and Boundary Layer Reentry Heating Techniques for Orbiter Nose Cap," Paper AIAA-86-1350 presented at AIAA/ASME $4^{\text {th }}$ Joint Thermophysics and Heat Transfer Conference in Boston, MA, June 2-4, 1986.

${ }^{9}$ Ting, P. C., Rochelle, W. C., and Curry, D. M., "Prediction of Aerodynamic Heating and Pressures on Shuttle Entry Air Data System (SEADS) Nose Cap and Comparison with STS-61C Flight Data," Paper presented at First International Conference on Hypersonic Flight in the $21^{\text {st }}$ Century, Grand Forks, ND, Sept. 20-23, 1988.

${ }^{10}$ Rakich, J. V., Stewart, D. A., and Lanfranco, M. J., "Catalytic Efficiency of the Space Shuttle Heat Shield," AIAA Progress in Astronautics and Aeronautics, Vol. 85, AIAA, Inc., 1983, pp. 97-122.

${ }^{11}$ Marichalar, J.J and Rochelle, W.C., "Catalytic Heating Factors as Derived from STS-2, STS-3, and STS-5 Orbiter Experiments (OEX) Flight Data," JSC-62579, April 2004

${ }^{12}$ Stewart, D. A., Rakich, J. V., and Lanfranco, M. J., "Catalytic Surface Effects of Space Shuttle Thermal Protection System During Earth Entry of Flights STS-2 Through STS-5," Shuttle Performance: Lessons Learned, NASA CP-2283, 1983, pp. 827-845.

${ }^{13}$ Scott, C. D., "A Review of Non-equilibrium Effects and Surface Catalysis on Shuttle Heating," Shuttle Performance: Lessons Learned, NASA CP-2283, 1983, pp. 865-889.

${ }^{14}$ Stewart, D. A., Rakich, J. V., and Chen, Y. K. "Flight Experiment Demonstrating the Effect of Surface Catalysis on the Heating Distribution over the Space Shuttle Heat Shield," Orbiter Experiments (OEX) Aerothermodynamics Symposium, NASA CP-3248, Part 2, April 1995.

${ }^{15}$ Scott, C. D. and Derry, S. M., "Catalytic Recombination/Space Shuttle Heating," AIAA Progress in Astronautics and Aeronautics, Vol. 85, AIAA, Inc., 1983, pp. 123-148.

${ }^{16}$ Bouslog, S. A., "Release of Local Heating Environments for RCC Repair, Rev. 2," NASA JSC memo, April 7, 2004 and presented on April 1, 2004 at Entry Aeroheating Panel Meeting

${ }^{17}$ Curry, D. M., Rodriguez, A., and Fields, M., "RCC Damage Assessment Status," presentation at Design Certification Review, Feb. 11, 2005.

${ }^{18}$ Rochelle, W. C. and Norman, I., "Review of Arc-Jet Aerothermal Environments," Rockwell International SE-TCS-95-099, November 13, 1995.

${ }^{19}$ Reynolds, D., Norman, I., Reyna, J., and Chao, D. C., "Plasma Arc Jet Test Results of Uncoated AVTEX and ENKA Reinforced Carbon-Carbon (RCC) for Transoceanic Abort (TAL) Landing Verification," Rockwell International SE-TSAT-90101, December 14, 1990.

${ }^{20}$ Norman, I., Chao, D. C., and Reyna, J., "Plasma Arc Tests for RCC TAL Abort Verification Coated AVTEX Reinforced Carbon-Carbon (RCC) Results," Rockwell International SE-TSAT-90-038, June 12, 1990.

${ }^{21}$ Reynolds, D., Norman, I., Reyna, J., and Chao, D. C., "Plasma Arc Jet Test results of Coated AVTEX and ENKA Reinforced Carbon-Carbon (RCC) for Transoceanic Abort Landing (TAL) Verification Phase II," Rockwell International SETSAT-91-005, February 15, 1991.

${ }^{22}$ Marichalar, J. J. and Rochelle, W. C., "Recommended Catalytic Heating Factors for Nose Region Damage Assessment during Orbiter Reentry," JSC Orbiter Entry Aeroheating Note OEAN 0305-002.A, April 6, 2005.

${ }^{23}$ Scott, C. D., "Wall Catalytic Recombination and Boundary Conditions in Non-equilibrium Hypersonic Flow - with Applications," presented at The Second Joint Europe/US Short Course in Hypersonics, U.S. Air Force Academy, Colorado Springs, CO, Jan. 17, 1989.

${ }^{24}$ Scott, C. D., "Catalytic Recombination of Nitrogen and Oxygen on High-Temperature Reusable Surface Insulation," AIAA Paper 80-1477 presented at $15^{\text {th }}$ Thermophysics Conference, Snowmass, CO, July 14-16, 1980.

${ }^{25}$ Stewart, D. A., "Surface Catalysis and Characterization of Proposed Candidate TPS for Access-to-Space Vehicles," NASA Technical Memorandum 112206, July 1997.

${ }^{26}$ Cheatwood, F. M. and Gnoffo, P. A., "User's Manual for the Langley Aerothermodynamic Upwind Relaxation Algorithm (LAURA)," NASA Technical Memorandum 4674, April 1996.

${ }^{27}$ McGrory, W. D., Stack, D. C., Applebaum, M. P., and Walthers, R. W., "GASP Version 3.1 - The General Aerodynamic Simulation Program," AeroSoft, Inc., August 1997.

${ }^{28}$ Marichalar, J. J. and Rochelle, W. C., "Summary of BLIMPK Catalytic Heating Analysis on Orbiter Lower Fuselage during STS-2, STS-3, and STS-5 Reentry," Presentation to Aeroheating Working Group, NASA JSC, Aug. 16, 2004.

${ }^{29}$ Tam, L. T., "LU-SGS Implicit Scheme for Entry Vehicle Flow Computation and Comparison with Aerodynamic Data," Paper AIAA-92-2671-CP presented at AIAA 10 ${ }^{\text {th }}$ Applied Aerodynamics Conference, Palo Alto, CA, June 22-24, 1992. 
${ }^{30}$ An, M. Y., Wang, K. C., and Tam, L. T., "Computation of Inviscid Flowfield Around 3-D Aerospace Vehicles and Comparison with Experimental \& Flight Data," AIAA Paper 93-0885, Reno, NV, January 11-14, 1993.

${ }^{31}$ Wang, K. C., "An Axisymmetric Analog Two-Layer Convective Heating Procedure with Applications to Evaluation of Space Shuttle Wing Leading Edge and Windward Surface Heating," NASA CR-188343, 1994.

${ }^{32}$ Kirk, B., "A Tool to Extract Streamline Data from Navier-Stokes Solutions for Boundary-Layer Solutions," in preparation, 2006.

${ }^{33}$ Wright, M. J., Candler, G. V. and Bose, D., "Data-Parallel Line Relaxation Method for Navier-Stokes Equations," AIAA Journal, Vol. 36, No. 9, Sept. 1998.

${ }^{34}$ S. Alter and J. Reuther, "New Common Mesh Development In Support of RTF," Presentation to RTF Aeroheating Working Group, December, 2004.

${ }^{35}$ J. Reuther, R. McDaniel, J. Brown, D. Prabhu, D. Saunders, and G. Palmer, "External Computational Aerothermodynamic Analysis of the Space Shuttle Orbiter at STS-107 Flight Conditions," Paper AIAA-2004-2281 presented at 37th AIAA Thermophysics Conference, Portland, Oregon, June 28-1, 2004.

${ }^{36}$ Saunders, D., "Curvature-Based Boundary Layer Edge Detection," in preparation, 2006.

${ }^{37}$ J. C. Tannehill, P. H. Mugge, "Improved curve fits for the thermodynamic properties of equilibrium air suitable for numerical computation using time-dependent or shock-capturing methods," NASA Tech. Rep. CR-2470, October, 1974.

${ }^{38}$ Rochelle, W. C. and An, M. Y., "Leading Edge Structural Subsystem (LESS) Entry Heating Study - Final Report. Vol. I - Methodology and Results," LESC-30453, May 1993.

${ }^{39}$ Scott, C. D., "Thermochemistry, Non-equilibrium, and Catalysis in Hypersonic Flows," Notes for a Short Course on Hypersonics at the University of Texas at Austin, April 1987.

${ }^{40}$ Marichalar, J., and Rochelle, B., "Summary of Recommended Catalytic Heating Factors for Orbiter Nose Region Damage Assessment During Reentry," Presentation to Aeroheating Panel, April 2005.

${ }^{41}$ Marichalar, J. J. and Fredo, J. A., "BLIMPK Catalytic Surface Heating Analysis on the Orbiter Wing Tile and RCC Wing Leading Edge," JSC-63232, August 2005. 